\title{
Microscale Complexity in the Ocean: Turbulence, Intermittency and Plankton Life
}

\author{
L. Seuront ${ }^{a, b 1}$ \\ ${ }^{a}$ School of Biological Sciences, Flinders University, GPO Box 2100, Adelaide 5001, Australia \\ ${ }^{b}$ South Australian Research and Development Institute, Aquatic Sciences \\ West Beach SA 5022, Australia
}

\begin{abstract}
This contribution reviews the nonlinear stochastic properties of turbulent velocity and passive scalar intermittent fluctuations in Eulerian and Lagrangian turbulence. These properties are illustrated with original data sets of (i) velocity fluctuations collected in the field and in the laboratory, and (ii) temperature, salinity and in vivo fluorescence (a proxy of phytoplankton biomass, i.e. unicelled vegetals passively advected by turbulence) sampled from highly turbulent coastal waters. The strength of three of the most popular models describing intermittent fluctuations (the lognormal, log-Lévy and log-Poisson models) to fit the distribution of in vivo fluorescence has subsequently been critically assessed. A theoretical formulation for the stochastic properties of biologically active scalars is also provided and validated. Finally, the potential effect of the intermittent properties of turbulent velocity fluctuations on processes relevant to the life of plankton organisms are theoretically investigated. It is shown that the intermittent nature of microscale turbulence may result in (i) a decrease in the rate of nutrient fluxes towards non-motile phytoplankton cells (6-62\%), (ii) a decrease in the physical coagulation of phytoplankton cells (25-48\%) and in the subsequent phytoplankton aggregate volumes (22-41\%), and (iii) a decrease of the turbulence contribution to predator-prey encounter rates (25-50\%).
\end{abstract}

Key words: patchiness, intermittency, plankton, Eulerian and Lagrangian turbulence AMS subject classification: 92B05, 92D25, 92D40

\footnotetext{
${ }^{1}$ E-mail: laurent.seuront@ flinders.edu.au
} 


\section{Introduction}

Numerous experimental data analyses done in different frameworks and geographical regions have shown that physical and biological patterns and processes in marine sciences display high intermittency [1-11]. This intermittency may correspond to a basic property of aquatic ecosystems, as sharp, local fluctuations are ubiquitously observed in space-time distributions of turbulent dissipation rates, temperature, salinity, nutrient and plankton concentration [3-7,10-12] and the motion behaviour of both benthic and pelagic organisms [13-15]. Practically, the occurrence of high density peaks positively skews a given probability distribution, causing it to deviate from Gaussianity (in which case the skewness is nil, and the probability distribution symmetric). In other words, intermittent variability reflects heterogeneous distributions with a few dense patches and a wide range of low-density patches (Fig. 1), and is hardly compatible with traditional statistical approaches. This form of variability has originally been described as log-normally distributed for fully developed turbulent flows $[16,17]$. The log-normal approach has, however, subsequently be severely criticized [18-21], stressing a critical need for a proper description of intermittent patterns [22].

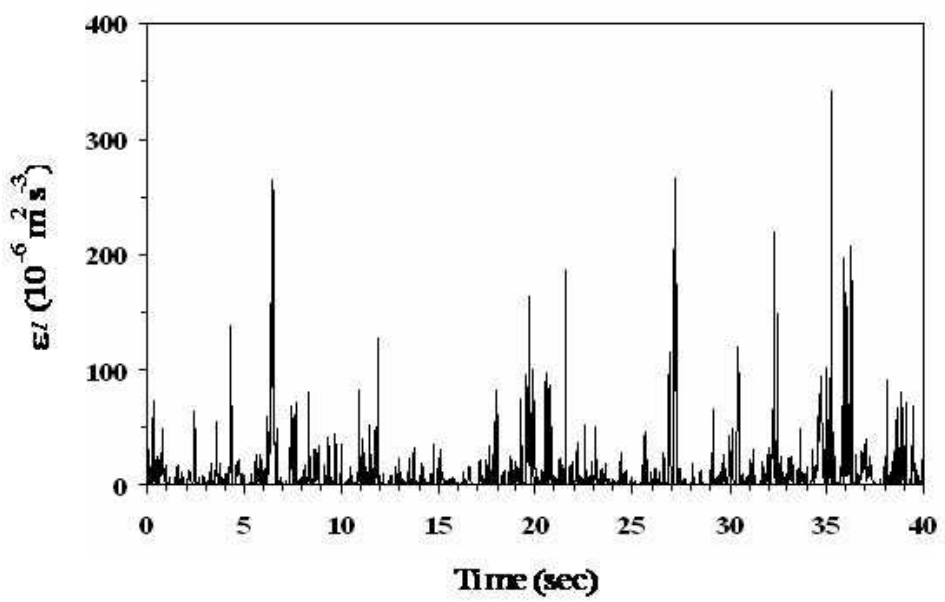

Figure 1: Instantaneous dissipation rates obtained from a grid-generated laboratory turbulence experiment where velocity fluctuations were recorded at $100 \mathrm{~Hz}$ with a hot-wire velocimeter, illustrating the concept of intermittency.

More specifically, if localized intermittent fluctuations have obvious practical implications in terms of sampling [1], they also have critical consequences on plankton biology and ecology, since planktonic mating, predator-prey contacts and chemical reactions all intrinsically occur at the ocean microscale (i.e. typically spatial scales smaller than $1 \mathrm{~m}$ ). It is thus crucial to accurately describe and model intermittency and the couplings between intermittent variables (i.e. turbulent velocity and phytoplankton abundance). In addition, as benthic and pelagic organisms experience their environment from Eulerian and Lagrangian perspectives (Fig. 2), these two frameworks need 
to be thoroughly investigated and understood to critically assess the impact of environmental fluctuations on their biology and ecology.

In this context, this contribution first provides a brief historical review of the use of intermittency in the literature, before discussing the related concepts found in the physical and biological literature and suggesting a definition of intermittent processes. A thorough description of the theoretical scaling properties for Eulerian and Lagrangian intermittent velocity and passive scalar fluctuations is then provided, and illustrated with original data for both physical and biological scalars. More specifically, the strength of three of the most popular models describing intermittent fluctuations (the lognormal, log-Lévy and log-Poisson models) to fit the distribution of in vivo fluorescence is critically assessed, and a theoretical formulation for the stochastic properties of biologically active scalars is provided and validated. The potential consequences of intermittent distributions for the ecology of marine organisms are finally theoretically investigated, and discussed in the general framework of their contribution to biogeochemical cycles.

\section{Intermittency}

\subsection{Historical perspectives}

The concept of intermittency (see Fig. 1 for an example of intermittent distribution) was originally introduced by Batchelor [23] who observed that "activation of large wavenumbers is very evenly distributed over space" and that "as the wavenumber is increased the fluctuations seem to tend to an approximate on-off, or intermittent, variation." Stewart [24] later specified that "The non-Gaussian, intermittent character of the small scale structure becomes more marked as the Reynolds number increases." It has also been acknowledged that while intermittency "seems to be fundamental to the nature of the turbulence cascade", "we do not have a fully satisfactory theoretical explanation" [24]. This limitation still stands today; the concept of intermittency, until very recently [25] has seldom been referred to and defined precisely, even in monographs devoted to turbulent processes [21,26-30]. In a 74-page chapter devoted to intermittency, Frisch [21] then only states that a process is intermittent when it "displays activity during only a fraction of the time, which decreases with the scale under consideration." Intermittency has similarly been described as "the active turbulent regions do not fill the whole volume, but only a subvolume in a very irregular way" [31] and "active regions occupy tiny fractions of the space available" [6].

\subsection{Intermittency in marine sciences}

Intermittency has seldom been described as such in marine sciences. In physical oceanography, intermittency has mainly been discussed in terms of its consequences on sampling, data processing and statistics $[1,2,20,32]$. The situation is similar in biological oceanography where turbulent intermittency and its potential effects are often not discussed. Patchiness, variability and heterogeneity - see [33] for terminological details - have been a major issue since the early studies [34-38]. Uneven plankton distributions have subsequently been widely described, e.g. [39-44]. However, intermittency has seldom been quantified, despite the increasing evidence of the intermittent nature 


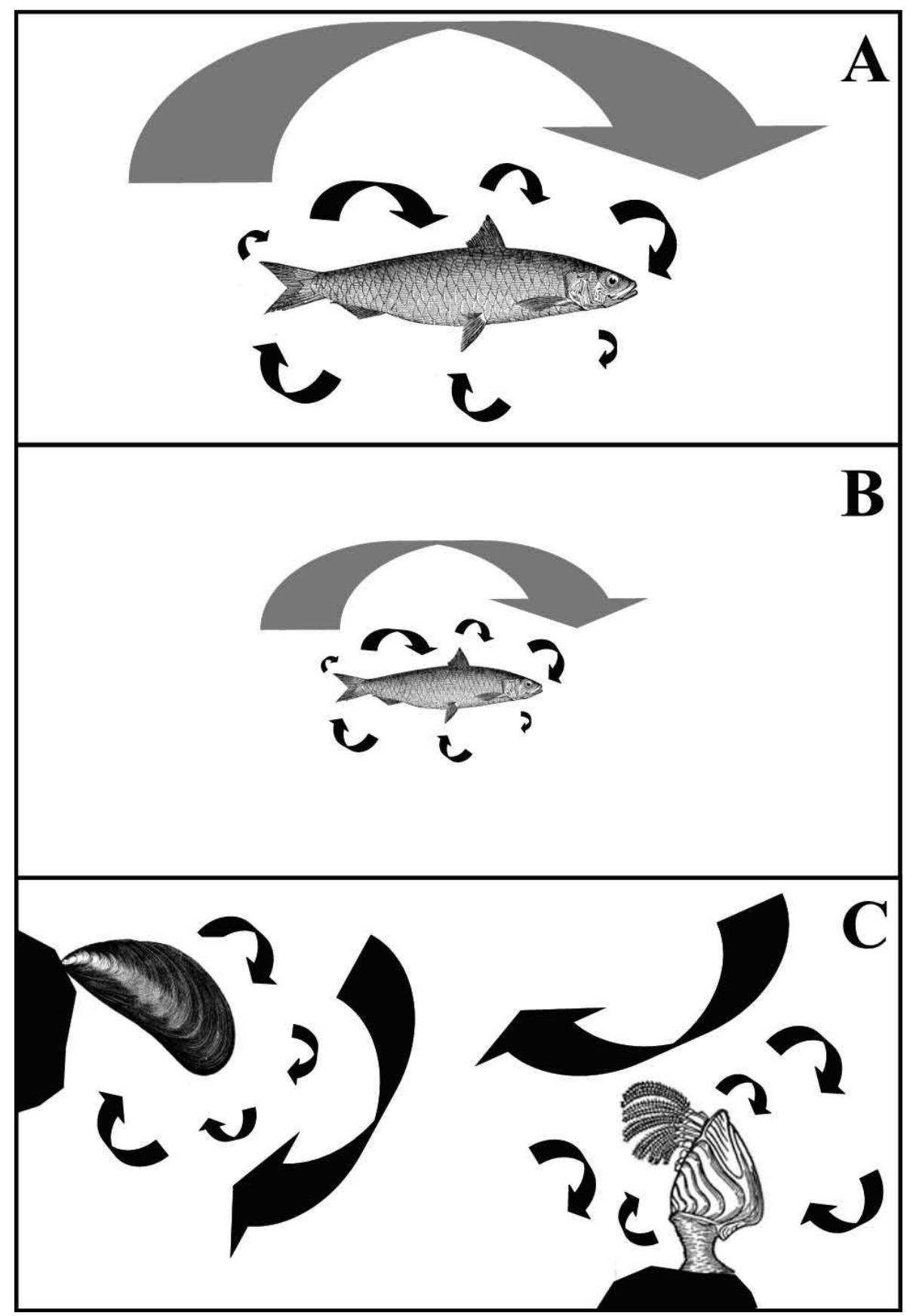

Figure 2: Illustration of Lagrangian and Eulerian perceptions of the environment by marine organisms. For swimming organisms $(a, b)$, the perception of their environment is intrinsically linked to their size. Velocity fluctuations larger than the organisms are then perceived in a Lagrangian way (grey arrows), while velocity fluctuations smaller than the organisms will be perceived in a Eulerian way (black arrows). In contrast sessile (i.e. fixed to a substrate) organisms always perceive their environment in a Eulerian way (black arrows). 
of plankton distributions [3-7,10-12]. Instead, turbulence intermittency has recurrently been considered as irrelevant to marine life. For instance, intermittent events have been described as "very intense from the point of view of plankton, but calculations show that their probability is small" [45]. Similarly, intermittent bursts "must certainly be spectacular events from the point of view of plankton, comparable to the passing of a tornado at our scale, and probably with similar consequences on the individual involved" but that "they are sufficiently rare that they can be neglected in most calculations" [31]. Intermittency has been widely observed; however, it has still escaped the confines of a narrow, precise definition.

\subsection{Towards a unified definition of intermittency}

The definition of intermittency greatly varies from author to author, and from field to field, leading to a largely scattered and non-unified framework. First, the phenomenon of intermittency has widely been mixed up with its statistical consequences, and thus generally poorly defined even in specialized monographs. The literature, hence, recurrently refers to intermittency through statements such as "the kurtosis is a useful measure of intermittency for signals having a bursty aspect" [21], "the signals tended to become bursty when the order of differentiation is increased" [21], "most of the time the gradients would still be of the order of magnitude of their standard deviation, but occasionally we should expect stronger bursts, more often than in the Gaussian case" [31], "the discrepancies between the Kolmogorov predictions and the experimental values of the high-order moments" [30], and "we occasionally should expect stronger bursts than expected in a non-intermittent, homogeneous turbulence, which accentuate the skewness of a given probability distribution, causing it to deviate from Gaussianity" [46].

The production of turbulence is not a continuous process but usually has an intermittent character and the turbulence appears as bursts [47]. This intermittency has been acknowledged as "a common phenomenon in many complex systems, and a natural consequence of cascades" [46]. Intermittency has also been related to the coherent nature of turbulence and the presence of strong vortices, with diameters of the order of 10 times the Kolmogorov length scale $l_{k}, l_{k}=\left(\nu^{3} / \epsilon\right)^{1 / 4}$ where $\nu$ is the kinematic viscosity $\left(\mathrm{m}^{2} \mathrm{~s}^{-1}\right)$ and $\epsilon$ the turbulent kinetic energy dissipation rate $\left(m^{2} s^{-3}\right)$ [31,45]. The term 'intermittency' has alternatively been coined to describe "the phenomena connected with the local variability of the dissipation" [47] as well as "instantaneous gradients of scalars such as temperature, salinity or nutrients, greatest at scales similar to the Kolmogorov microscale" [48]. Intermittent flows were also characterized as a motion that "is sometimes turbulent and sometimes non-turbulent" [30], while 'internal intermittency' has been used to characterize the strong fluctuations perceptible in the instantaneous distribution of the dissipation rate observed by Batchelor and Townsend [49]. A more intuitive definition stated that "this form of variability reflects heterogeneous distributions with a few dense patches and a wide range of low density patches" [46]. Finally, in the engineering community, an intermittent flow has been defined as a flow which is "sometimes laminar and sometimes turbulent" in reference to the behaviour of boundary layer free-stream velocity [50].

Most of the previously published work referred to intermittency in the framework of turbulent flows. However, a general consensus can be reached considering that a given pattern/process is 
intermittent in space or in time if (i) it is characterized by sharp fluctuations, (ii) it is responsible for a skewed probability distribution, and (iii) it has a long-term memory signature, perceptible from the power law form of its autocorrelation function.

\section{Eulerian turbulence and intermittency}

\subsection{Eulerian scaling relations for velocity and passive scalars}

This section reviews the rationale behind the range of scaling relations developed for Eulerian velocity and passive scalars fluctuations from the seminal work of Kolmogorov [51], hereafter referred to as K41, who introduced the ' $-5 / 3$ ' power law of the velocity spectrum of homogeneous in the initial subrange to the introduction of velocity structure functions to study the high order statistical properties of intermittent turbulence [52].

Scaling relations for turbulent velocity and passive scalar (originally temperature) fields have been expressed in Eulerian turbulence using the energy flux $\epsilon$ as [51,53]:

$$
\epsilon \approx \frac{\left(\Delta V_{l}\right)^{3}}{l}
$$

and the scalar variance flux $\chi$ as [50-52]:

$$
\chi \approx \frac{\left(\Delta S_{l}\right)^{2}\left(\Delta V_{l}\right)}{l}
$$

where $\Delta V_{l}=|V(x+l)-V(x)|$ and $\Delta S_{l}=|S(x+l)-S(x)|$ are the velocity shear and passive scalar gradients at scale $l$ and $\Delta V_{l} / l$ is the inverse of the local eddy turnover time. These scaling relations were originally considered in the framework of homogeneous turbulence, i.e. the fluxes $\epsilon$ and $\chi$ were considered as homogeneous, exhibiting no scale dependence. The statistics of turbulent velocity and passive scalar fluctuations were then regarded as universal, and determined by the mean dissipation rate and the mean scalar variance flux $\chi$. Consequently, a unique exponent was required for the velocity and passive scalar, the so-called 1/3 law in physical space:

$$
\begin{aligned}
& \Delta V_{l} \approx l^{1 / 3}, \\
& \Delta S_{l} \approx l^{1 / 3} .
\end{aligned}
$$

In Fourier space, assuming local isotropy and three-dimensional homogeneity of turbulence in the inertial subrange, Eqs. (3) and (4) can be rewritten to describe the velocity fluctuations and the fluctuations of a passive scalar using the spectral densities $E_{V}(k)$ and $E_{S}(k)$ as:

$$
\begin{aligned}
& E_{V}(k) \approx k^{-\beta_{V}}, \\
& E_{S}(k) \approx k^{-\beta_{S}},
\end{aligned}
$$


where $k$ is either the frequency $(\mathrm{Hz})$ or the wavenumber $\left(\mathrm{m}^{-1}\right)$ whether velocity and passive scalar fluctuations are considered in time or in space, and $\beta_{V}$ and $\beta_{S}$ are characteristic spectral exponents defined as $\beta_{V}=\beta_{S}=5 / 3$. This has been verified for velocity and temperature fluctuations in the atmosphere [54], in the ocean [4-6,10] and in laboratory experiments [25], and for phytoplankton biomass in a variety of marine environments [4-6,55,56].

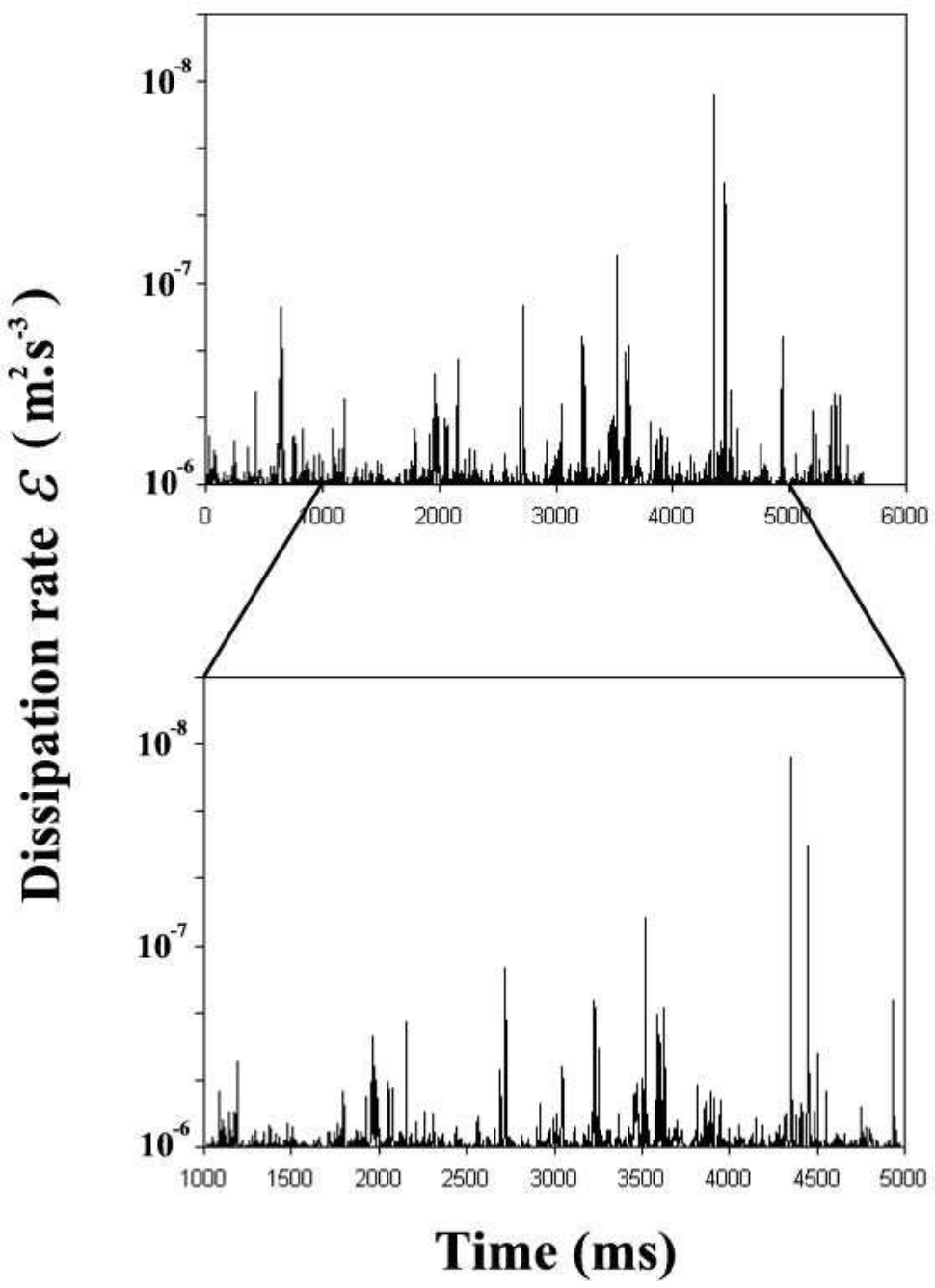

Figure 3: Nested structure perceptible in time series of microscale turbulent kinetic energy dissipation rates $\left(\mathrm{m}^{2} . \mathrm{s}^{-3}\right)$. At increasing resolution, the local and global structures remain very similar.

However, the flux of energy and the flux of scalar variance exhibit sharp intermittent fluctuations at all scales (Fig. 3). More specifically, Batchelor and Towsend [49] showed that instantaneous dissipation rates intermittently reach very high values and that this intermittency was more important when the scale ratio, hence the Reynolds number, is large. Here turbulent velocity and scalar fluctuations are intermittent in the sense that active regions occupy tiny fractions of the available space. The original assumption of homogeneity then becomes untenable and turbulent fields 
had to be thought as intermittent and scale-dependent processes. This led to the refined similarity hypothesis [16,17], stating that velocity fluctuations are influenced by the local value of the dissipation averaged over a distance $l, \epsilon_{l}$. The 'refined similarity hypothesis' [16,17], then leads to the introduction of the subscript $l$ in Eqs. (1) and (2) that now read:

$$
\epsilon_{l} \approx \frac{\left(\Delta V_{l}\right)^{3}}{l}
$$

and

$$
\chi_{l} \approx \frac{\left(\Delta S_{l}\right)^{2}\left(\Delta V_{l}\right)}{l}
$$

A log-normal distribution has been introduced for $\epsilon_{l}$ [16,17], leading to describe all the statistics of a turbulent field with only two parameters, the mean and the variance. However, since the use and the relevance of second order statistics can be restrictive and characterises intermittent fluctuations very poorly, the velocity structure functions were introduced in order to study the statistical properties of turbulence [52]. The $q^{\text {th }}$ order structure functions are an empirical generalisation of the widely used power spectral approach [52]:

$$
\left\langle\left|\Delta S_{l}\right|^{q}\right\rangle \approx l^{\zeta_{S}(q)}
$$

where $\Delta S_{l}$ is the fluctuations of a scalar field at scale $l$ and " \langle\rangle " means ensemble average, i.e. $\left\langle\left(\Delta S_{l}\right)^{q}\right\rangle$ is the statistical moments of the fluctuations, $\left(\Delta S_{l}\right)=\left(S_{x+l}-S_{x}\right)$. Eq. (9) gives the scale invariant structure functions' exponent $\zeta_{S}(q)$, which characterises all the statistics of the field. The first moment characterises the mean of the fluctuations: when non zero, it expresses the scale dependency of the amplitude of the fluctuations: $H=\zeta_{S}(1)$. For simple scaling (monofractal) processes such as Brownian motion, the scaling exponent of the structure function $\zeta_{S}(q)$ is linear. For multiscaling (i.e. intermittent) processes, this function is non-linear and convex. Figure 4 illustrates the structure function analysis of turbulent velocity fluctuations (Fig. 4a) and oceanic in vivo fluorescence fluctuations (Fig. 4d). Figures $4 \mathrm{~b}$ and e show the scaling of the structure functions for various order of moments: in a log-log plot, the straight lines provide estimates of $\zeta_{V}(q)$ and $\zeta_{S}(q)$. The functions $\zeta_{V}(q)$ and $\zeta_{S}(q)$ are clearly nonlinear and convex (Fig. 4c, f), and strongly diverge from the theoretical shapes $\zeta_{V}(q)=q / 3$ (Fig. 4c) and $\zeta_{S}(q)=q H$ (Fig. 4f). A critical point here is that the structure functions of turbulent velocity and passive scalar fluctuations are directly related to the PDF of the local dissipation rate $\epsilon_{l}$ and variance flux $\chi_{l}$. A proper model for the PDF of $\epsilon_{l}$ and $\chi_{l}$ is then sufficient to describe the whole statistics of turbulent velocity. The prediction of the original log-normal proposal is in reasonable agreement with empirical data for statistical moments $q$ of sufficiently low order (i.e. $q \leq 10)$. For large order $(q>10)$, the discrepancies are attributed to the deficiencies in the log-normal assumption, which has been severely criticized [18-21]. Another consequence of intermittency is the introduction of an intermittent correction to the Kolmogorov spectral slope, the intermittency exponent $\mu$ which is intrinsically linked to the distribution chosen for $\epsilon_{l}$ and $\chi_{l}$ [22]. This issue will be thoroughly investigated in Section 3.2.

In cascade models of turbulence, the intermittent fluxes $\epsilon_{l}$ and $\chi_{l}$ result from a multiplicative process in which the variability builds up from large to small scales. This leads to multifractal 

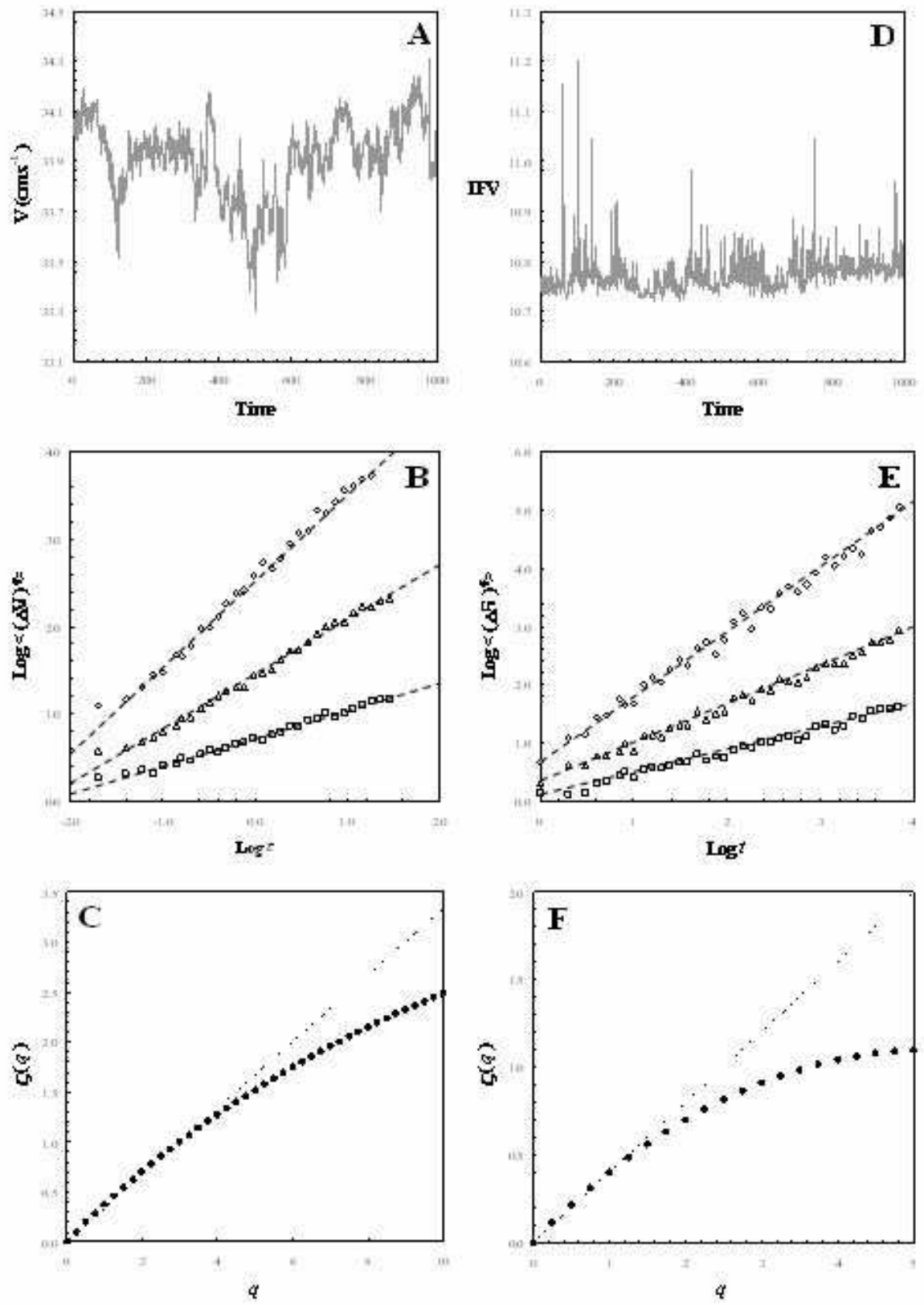

Figure 4: Time series of grid-generated turbulent velocity recorded by hot-wire velocimetry at 100 $\mathrm{Hz}$ in a circular flume (a) and in vivo fluorescence recorded in the coastal waters of the eastern English Channel (d). The corresponding structure function analyses are shown in (b) and (e) for different values of q (, 2 and 3 from bottom to top). The related structure functions exponents are clearly nonlinear and convex, illustrating the intermittent nature of velocity and fluorescence fluctuations $(\mathrm{c}, \mathrm{f})$. The dashed lines are the theoretical, non-intermittent exponents for turbulent velocity (c) and fluorescence (f). 
fields with [57]:

$$
\begin{gathered}
\left\langle\left(\epsilon_{l}\right)^{q}\right\rangle \approx \lambda^{K_{\epsilon}(q)} \approx l^{-K_{\epsilon}(q)}, \\
\left\langle\left(\chi_{l}\right)^{q}\right\rangle \approx \lambda^{K_{\chi}(q)} \approx l^{-K_{\chi}(q)}, \\
\left\langle\left|\Delta V_{l}\right|^{q}\right\rangle \approx \lambda^{-\zeta_{V}(q)} \approx l^{\zeta_{V}(q)}, \\
\left\langle\left|\left(\Delta S_{l}\right)^{2} \Delta V_{l}\right|^{q}\right\rangle \approx \lambda^{-\zeta_{V, S}(3 q)} \approx l^{\zeta_{V, S}(3 q)},
\end{gathered}
$$

where the angle brackets \langle\rangle indicate ensemble averaging, $\lambda$ the scale ratio between the largest external scale $L$ and the actual scale $l$ (i.e. $\lambda=L / l), K_{\epsilon}(q)$ and $K_{\chi}(q)$ the scaling moment functions for the fluxes $\epsilon_{l}$ and $\chi_{l}, \zeta_{V}(q)$ and $\zeta_{V, S}(q)$ the scaling moment functions of the velocity structure function and the joint structure function scaling exponent of the product $\left(\Delta S_{l}\right)^{2} \Delta V_{l}$. From Eqs. (3.7) and (3.8), the functions $K_{\epsilon}(q)$ and $K_{\chi}(q)$ can be defined as:

$$
\begin{gathered}
K_{\epsilon}(q)=q-\zeta_{V}(3 q), \\
K_{\chi}(q)=q-\zeta_{V, S}(3 q) .
\end{gathered}
$$

Because the fluxes are conserved by the equation of motion over the inertial subrange, they are assumed to be scale-independent:

$$
\begin{aligned}
& \left\langle\epsilon_{l}\right\rangle \approx\left\langle\epsilon_{1}\right\rangle, \\
& \left\langle\chi_{l}\right\rangle \approx\left\langle\chi_{1}\right\rangle .
\end{aligned}
$$

Eqs. (3.10) and (3.11) subsequently lead to:

$$
\begin{aligned}
& K_{\epsilon}(1)=0, \\
& K_{\chi}(1)=0 .
\end{aligned}
$$

Such multifractal fields are called "conservative multifractals", and the conservation of the fluxes $\epsilon_{l}$ and $\chi_{l}$ (Eqs. (3.16) and (3.17)) leads to:

$$
\begin{gathered}
\zeta_{V}(3)=1, \\
\zeta_{V, S}(3)=1 .
\end{gathered}
$$

Eqs. (3.20) and (3.21) correspond to the exact formulations for the small-scale dissipation fields [51,58]. The scaling moment functions $K_{\epsilon}(q), K_{\chi}(q), \zeta_{V}(q)$ and $\zeta_{V, S}(q)$ characterize all the fluctuations of the fluxes of energy and scalar variance, and the fluctuations of the velocity shear and 
scalar gradient. In other words, as under fairly general conditions the probability distribution of a random variable is equivalently specified by its statistical moments, the scaling moment functions $K(q)$ and $\zeta(q)$ describe the scale dependence of the statistical moments of order $q$.
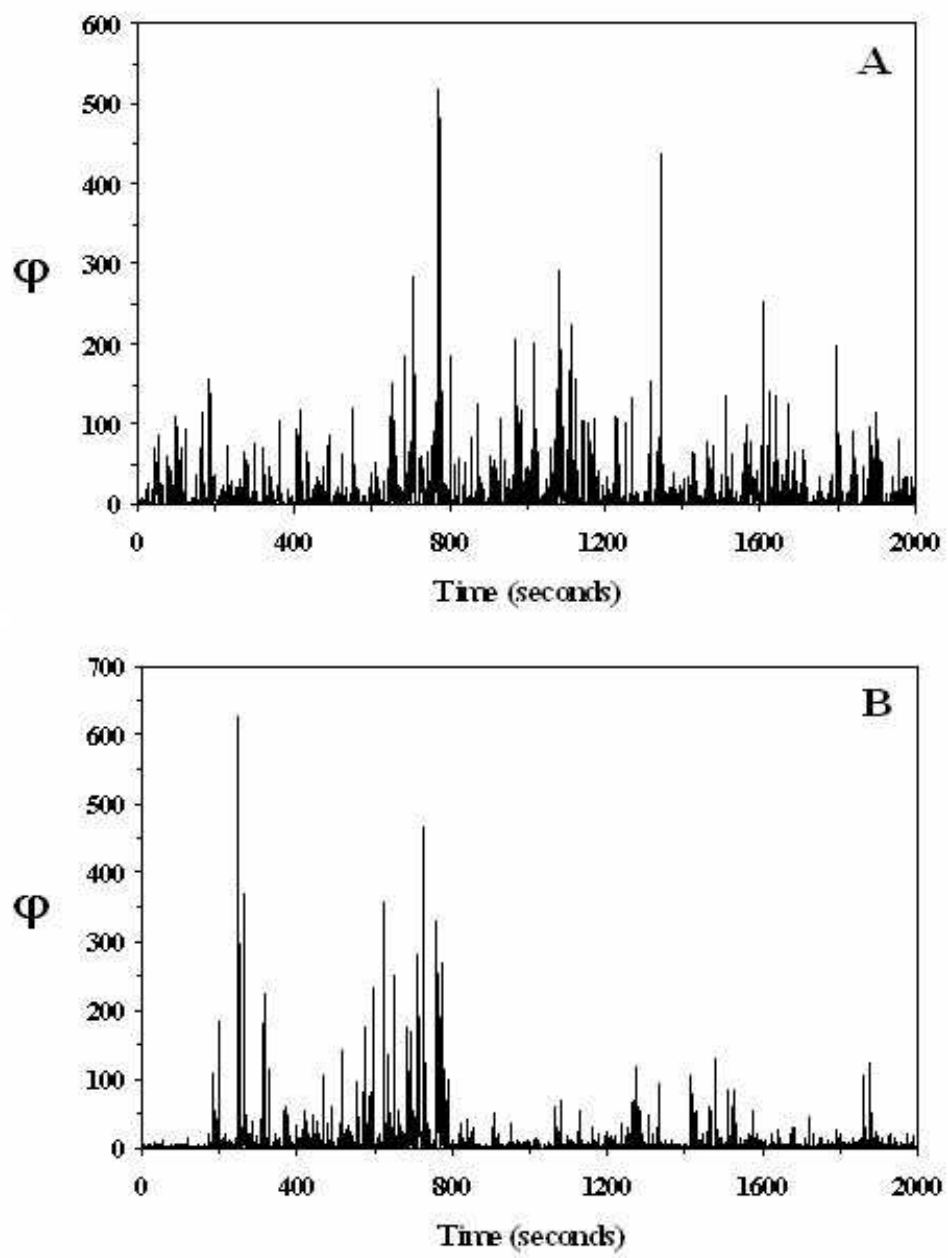

Figure 5: Time series of the rates of variance fluxes $\phi_{l}$ estimated from in vivo fluorescence (fluorescence ${ }^{2} s^{-1}$; a) and temperature $\left(\times 10^{-6}{ }^{0} C^{2} s^{-1}\right.$; b) recorded in the eastern English Channel with a Sea Tech fluorometer and a Sea-Bird Sealogger CTD, respectively.

Eqs. (3.10) to (3.13) characterise all the fluctuations of the energy flux $\epsilon_{l}$ (Eq. (3.7)) and scalar variance flux $\chi_{l}$ (Eq. (3.8)) through the scaling moment functions $K(q)$ and $\zeta(q)$. The fluctuations of a passive scalar are defined by the scaling moment function $\zeta_{S}(q)$ as:

$$
\left\langle\left|\Delta S_{l}\right|^{q}\right\rangle \approx \lambda^{-\zeta_{S}(q)}
$$

However, because the passive scalar flux $\phi_{l}$ (Fig. 5) is a non-conservative mixed flux of energy $\epsilon_{l}$ and scalar variance $\chi_{l}$ as: 


$$
\phi_{l}=\epsilon_{l}^{-1 / 2} \chi_{l}^{3 / 2},
$$

which are intrinsically correlated, the scaling moment function $\zeta_{S}(q)$ is related to the structure function of velocity fluctuations and scalar gradients as:

$$
\zeta_{S}(q)=\zeta_{V, S}(3 q / 2)-\zeta_{V}(q / 2),
$$

where $\zeta_{V}(q)=q / 3+K_{\epsilon}(q / 6)-K_{\chi}(q / 2)$; see [58]. Eqs. (3.8) and (3.23) also lead to expressed the squared passive scalar fluctuations, $\left\langle\Delta S_{l}^{2}\right\rangle=\left\langle\left(S_{x+l}-S_{x}\right)^{2}\right\rangle$, as:

$$
\left\langle\Delta S_{l}^{2}\right\rangle \approx \phi_{l}^{2 / 3} l^{2 / 3}
$$

With the introduction of discrete and continuous cascade models, a wide variety of distributions (including improvement of the initial log-normal proposal) has been proposed for $\epsilon_{l}$ and $\phi_{l}$. A brief review of the distributions found in the literature, and a test of their performance at fitting oceanic in vivo fluorescence fluctuations (i.e. a proxy for phytoplankton biomass) are proposed in Section 3.2 .

\subsection{Cascade models for turbulent intermittency}

\subsubsection{Background}

The first description of the turbulence cascade came with the intuitive scheme of Richardson [59], who recognized that "big whirls have little whirls that feed on their velocity, and little whirls have lesser whirls and so on to viscosity in the molecular sense" (Fig. 6). This was later formalized by the self-similarity hypothesis [51] which states that velocity fluctuations between two points separated by a distance $l$ depend only on the average dissipation rate $\epsilon$. The squared velocity fluctuations, $\left\langle\Delta V_{l}^{2}\right\rangle=\left\langle\left(V_{x+l}-V_{x}\right)^{2}\right\rangle$ thus writes:

$$
\left\langle\Delta V_{l}^{2}\right\rangle \approx \epsilon^{2 / 3} l^{2 / 3}
$$

where $x$ and $x+l$ are two points separated by a distance $l$. In Fourier space Eq. (3.26) is strictly equivalent to Eq. (3.5) for turbulent velocity fluctuations and Eq. (3.25) to Eq. (3.6) for passive scalar fluctuations. The generalized structure functions for moments of order $q>0$ of the absolute velocity increments are defined as:

$$
\left\langle\left|\Delta V_{l}\right|^{q}\right\rangle \approx \epsilon^{q / 3} l^{q / 3}
$$

Eq. (3.27) leads to the K41 linear law (i.e. non-intermittent, homogeneous turbulence):

$$
\zeta_{V}(q)=q / 3
$$

where $\zeta_{V}(q)$ is the scaling exponent of the velocity structure functions:

$$
\left\langle\left|\Delta V_{l}\right|^{q}\right\rangle \approx l^{\zeta_{V}(q)}
$$


For any passive scalar advected by turbulent flows, Eqs. (3.25) rewrite as:

$$
\left\langle\left|\Delta S_{l}\right|^{q}\right\rangle \approx l^{\zeta_{S}(q)}
$$

where $\zeta_{S}(q)$ is the scaling moment function of scalar gradients; see Eq. (3.24). Furthermore, Eqs. (3.26) and (3.27) lead to a general relationship between the spectral exponent $\beta$ and the secondorder moment structure function exponent $\zeta(2)$ :

$$
\beta_{i}=1+\zeta_{i}(2),
$$

where $\beta_{i}=\beta_{V}$ for velocity fluctuations (see Eq. (3.5)) and for passive scalar fluctuations (see Eq. (3.5)), and $\zeta_{i}(2)=\zeta_{V}(2)$ for velocity fluctuations (see Eq. (3.29)) and $\zeta_{i}(2)=\zeta_{S}(2)$ for passive scalar fluctuations; see Eq. (3.9).

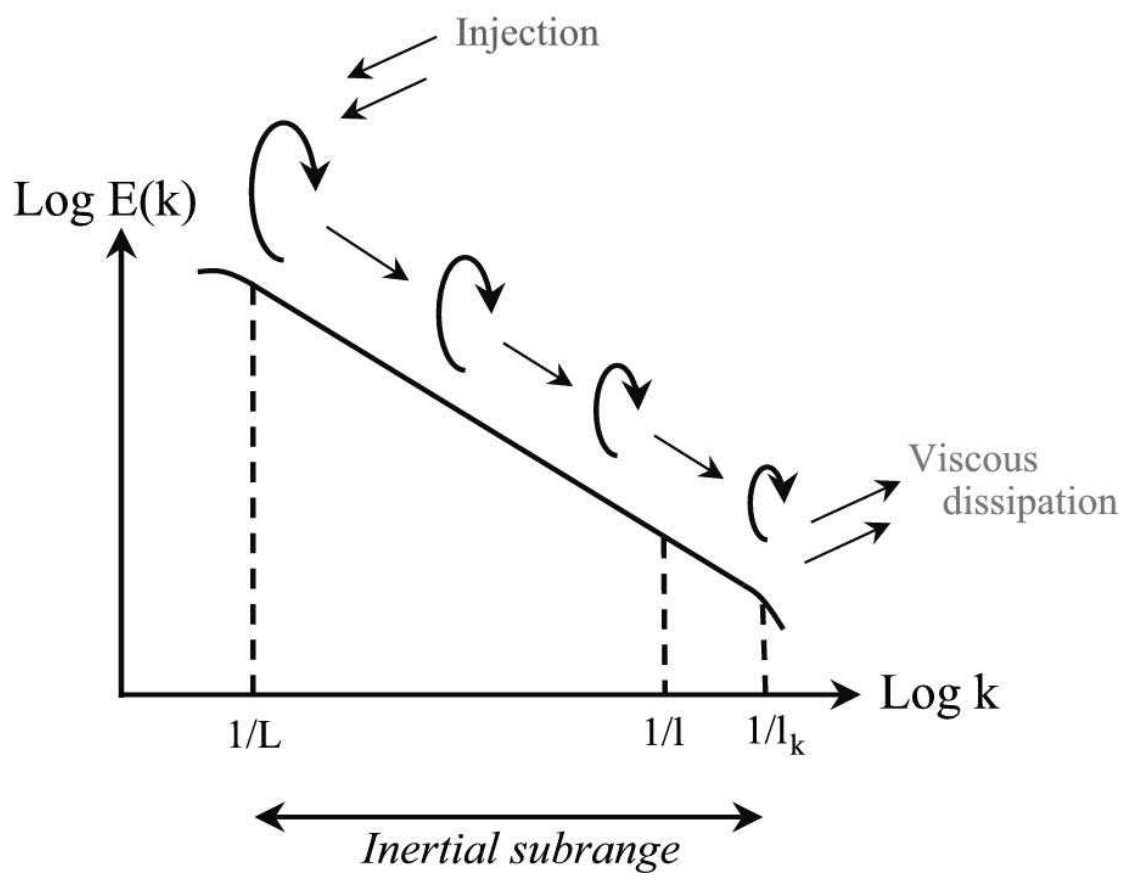

Figure 6: Schematic illustration of the energy spectrum of turbulent velocity cascade, where $E(k)$ is the spectral density and $k$ is a wavenumber $\left(m^{-1}\right)$ or a frequency $(\mathrm{Hz})$ whether turbulence is considered in space or in time. The kinetic energy generated at large scale $L$ cascades through the inertial subrange through a hierarchy of eddies of decreasing size down to the viscous Kolmogorov scale $l_{k}$, where it is dissipated into heat by viscous effects.

Kolmogorov [17] and Obukhov [16] took intermittency into account considering that the structure function of velocity fluctuations is a function of a locally averaged dissipation rate for a sphere of radius $l, \epsilon_{l}$. They hypothesized that $\epsilon_{l}$ was $\log$-normally distributed with the variance $\sigma_{l}^{2}$ of $\log \epsilon_{l}$ given by:

$$
\sigma_{l}^{2}=A+\mu \log (L / l)
$$


where $A$ is a constant associated with the macrostructure of the flow, $\mu$ a universal constant, and the largest external scale. This hypothesis has been introduced without firm theoretical foundations; Kolmogorov [17] did not provide any justification for this hypothesis, just stating that "it is natural to suppose that". Obukhov [16] was more specific, indicating that the distribution of any positive quantity should be approximated by a log-normal distribution with the appropriate values for the first and second moments. For velocity fluctuations, Eq. (3.27) can be rewritten as:

$$
\left\langle\left|\Delta V_{l}\right|^{q}\right\rangle \approx \epsilon_{l}^{q / 3} l^{q / 3}
$$

Eq. (3.33) is also known as the refined similarity hypothesis (RSH). This leads to nonlinear forms for the structure function exponent $\zeta_{V}(q)$, characterizing intermittency.

\subsubsection{Cascade models for turbulence}

Since the first attempt to provide a quantitative description of the Richardson cascade was made by Yaglom [60] and Gurvich and Yaglom [61], a range of discrete and continuous cascade models have been introduced to describe intermittent fluxes; see [22] for a review.

A first family of models is composed of discrete models, for which the scale ratio between a structure and the daughter structure is a discrete integer. Due to their discrete nature, these models are not realistic, but have been introduced for their simplicity and ability to reproduce experimental intermittency. These models include the log-normal model, the mono-fractal $\beta$-model, the $\alpha$ model, the $p$-model, the random $\beta$-model and the $B$-model. A detailed review of these models may be found in $[21,22,62,63]$. The limitations of these models and their limited ability to fit experimental data, especially for the higher orders of moment $q$, are detailed in [21].

The continuous log-infinitely divisible (log-ID) stochastic models represent a more realistic family of cascade models. Specifically, infinite divisibility specifies that any random variable belonging to this law may be written as a sum of an arbitrarily large number of independent random variables having each the same law (independent identically distributed; see e.g., [64]). This property considerably restricts available probability laws; the most known ID laws are the Gaussian, Lévy-stable, Poisson and Gamma. We review here the corresponding log-ID continuous cascade models for velocity fluctuations and passive scalar fluctuations, and evaluate their ability to fit the fluctuations of oceanic in vivo fluorescence (i.e. a proxy of phytoplankton biomass).

The lognormal model [16,17] corresponds to a quadratic form for $\zeta_{V}(q)$ and $\zeta_{S}(q)$. For velocity fluctuations, Eq. (3.20) and the condition lead to express $\zeta_{V}(q)$ as:

$$
\zeta_{V}(q)=\frac{q}{3}-\frac{\mu}{2}\left(\left(\frac{q}{3}\right)^{2}-\frac{q}{3}\right)
$$

where the intermittency parameter $\mu=K_{\epsilon}(2)$, and $K_{\epsilon}(q)=\frac{\mu}{2}\left((q / 3)^{2}-(q / 3)\right)$ (Fig. 7a). Similarly, for passive scalar fluctuations, the function $\zeta_{S}(q)$ writes as:

$$
\zeta_{S}(q)=q H-\frac{\mu}{2}\left(q^{2}-q\right),
$$

where the parameter $H$ is the degree of non-conservation of the average field ( $H=\zeta_{S}(1), \mu=$ $K_{\phi}(2)$ and $\left(K_{\phi}(q)=\frac{\mu}{2}\left(q^{2}-q\right)\right)$ (Fig. 7b). 

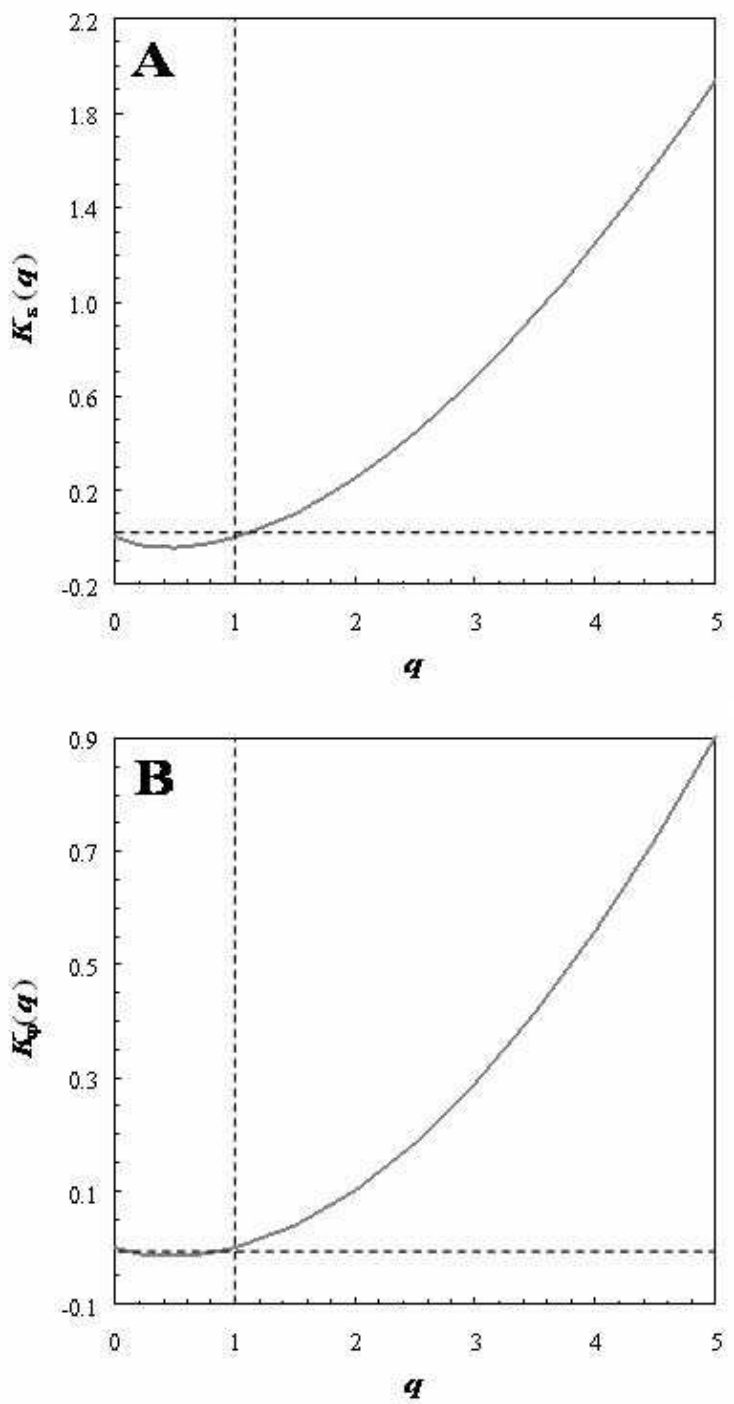

Figure 7: The intermittent functions $K_{\epsilon}(q)$ and $K_{\phi}(q)$ estimated from the turbulent velocity and in vivo fluorescence time series presented respectively in Fig. 4a and 4d. 
Because the Gaussian law belongs to the Lévy-stable family of random distributions, i.e. stable and attractive processes under addition [64], the lognormal cascade model has been generalized to log-stable cascades $[65,66]$. In this context, the structure function scaling exponents $\zeta(q)$ have a precise theoretical shape defined as [5]:

$$
\zeta(q)=A q+B q^{\alpha},
$$

where $A$ and $B$ are empirical constants, and $\alpha$ is the Lévy exponent for stable variables [64]. Eq. (3.36) defines a family of distributions defined according to the value of $\alpha, 0<\alpha \leq 2$. When $\alpha=2$, Eq. (3.36) recovers the lognormal model, and the $\beta$-model when $\alpha \rightarrow 0$ [22]. For $1 \leq \alpha<2$, the scaling is super-diffusive [67], while the value $\alpha=1$ indicates that the scaling becomes quadratic in time, and corresponds to the lower limit of super-diffusive processes, i.e. Lévy flight [67]. In contrast, values $\alpha \leq 0$ do not correspond to probability distributions that can be normalized. When $\alpha \leq 2$, the variance of the process diverges, and when $\alpha \leq 1$ the mean is not defined [15]. For turbulent velocity, the normalization condition $\zeta_{V}(3)=0$ leads to reformulate Eq. (3.34) as:

$$
\zeta_{V}(q)=\frac{q}{3}-\frac{C_{1 \epsilon}}{\alpha_{\epsilon}-1}\left(\left(\frac{q}{3}\right)^{\alpha_{\epsilon}}-\frac{q}{3}\right)
$$

where $K_{\epsilon}(q)=\frac{C_{1 \epsilon}}{\alpha_{\epsilon}-1}\left(\left(\frac{q}{3}\right)^{\alpha_{\epsilon}}-\frac{q}{3}\right)$, and the parameter $C_{1 \epsilon}\left(C_{1 \epsilon}=\mu / 2\right)$ is an intermittency parameter characterizing the fractal dimension of the mean. It satisfies $0 \leq C_{1 \epsilon} \leq 1$ (the larger $C_{1 \epsilon}$, the more intermittent is the process): $C_{1 \epsilon}=0$ for a homogeneous process and $C_{1 \epsilon}=0$ for a process so heterogeneous that the fractal dimension of the set contributing to the mean is zero [6]. For passive scalar fluctuations, the lack of know condition of normalization leads to:

$$
\zeta_{S}(q)=q H-\frac{C_{1 \phi}}{\alpha_{\phi}-1}\left(q^{\alpha_{\phi}}-q\right)
$$

where $K_{\phi}(q)=\frac{C_{1 \phi}}{\alpha_{\phi}-1}\left(q^{\alpha_{\phi}}-q\right)$. We can estimate $C_{1 \phi}$ and $\alpha_{\phi}$ from Eq. (3.38): if Eq. (3.38) is differentiated and evaluated at $q=0$, simple algebra shows that:

$$
q \zeta^{\prime}(0)-\zeta(q)=\frac{C_{1 \phi} q^{\alpha}}{\alpha_{\phi}-1}
$$

is given by the slope of $\left(q \zeta^{\prime}(0)-\zeta(q)\right)$ vs. $q$ in a log-log plot, and $C_{1 \phi}$ can be estimated from the intercept.

The log-Poisson model [68-71] describes the functions $\zeta_{V}(q)$ and $\zeta_{S}(q)$ as:

$$
\zeta_{V}(q)=\frac{q}{3}-c\left((1-\gamma) \frac{q}{3}-1+\gamma^{q / 3}\right)
$$

and

$$
\zeta_{S}(q)=q H-c\left((1-\gamma) q-1+\gamma^{q}\right),
$$

where $K_{\epsilon}(q)=c\left((1-\gamma) \frac{q}{3}-1+\gamma^{q / 3}\right)$ and $K_{\phi}(q)=c\left((1-\gamma) q-1+\gamma^{q}\right), \gamma$ is linked to a maximum intermittency (i.e. the most extreme event reachable from a finite sampling), and $c$ is 
an analogue of $C_{1}$ and characterizes the heterogeneity of this maximum intermittency. To estimate the parameters $\gamma$ and $c$, one needs to note that

$$
\zeta_{S}^{\prime}(q+1)=\left(1-\frac{\gamma}{c}\right) \zeta_{S}^{\prime}(q)+\frac{\gamma}{c}(H-\gamma)
$$

The slope and the intercept of a $\zeta^{\prime}(q+1)$ vs. $\zeta^{\prime}(q)$ plot give respectively $(1-\gamma / c)$ and $\frac{\gamma}{c}(H-\gamma)$, then considering the parameter $H$ (i.e. $H=\zeta(1)$ ), the estimation of $\gamma$ and $c$ is straightforward.

\subsubsection{Evaluation of cascade models for passive scalars}

The strength of the lognormal (LN), log-Lévy (LL) and log-Poisson (LP) models to describe the intermittent properties of passive scalars passively advected by fully developed turbulence was assessed against the functions $\zeta_{S}(q)$ estimated from a time series of temperature sampled simultaneously to the in vivo fluorescence time series shown in Fig. 4. Figure 8 shows the function $\zeta_{S}(q)$, for moment up to order 8 (with a 0.1 increment). The reader is referred to [22] for an evaluation of continuous cascade models to characterize the intermittency of turbulent velocity fluctuations in the atmosphere and the ocean. The theoretical fits given by Eqs. (3.35), (3.38) and (3.41), for the lognormal, log-Lévy and log-Poisson, with the values proposed in Table 1, are shown on the same figure. The lognormal model fits the empirical data for statistical moments $q<25$, indicating that it is compatible with the data up to relatively low order of moments. In contrast, the empirical curve and both the log-Lévy and log-Poisson models are indistinguishable for moments up to order 3 to 5 (Fig. 8). The scaling exponents $\zeta_{S}(q)$ were linear after a critical moment $q_{c}$ because of sampling limitations; see [6] for further details on multifractal phase transitions. Phase transitions are associated with the occurrence of a maximum intermittency $\gamma_{\max }$. In the case of first-order phase transition, $\gamma_{\max }$ is the maximum values taken by a given scalar associated with the occurrence of very rare and violent intermittencies. In the case of a second-order phase transition, $\gamma_{\max }$ corresponds to the maximum intermittency effectively detected from a finite sample size. In both cases, for $q \geq q_{c}$, the function $\zeta_{S}(q)$ has the following linear asymptotic behaviour:

$$
\zeta_{S}(q)=1-\gamma_{\max } q
$$

In the case of sampling limitations, the critical exponent $q_{s}$ is given by:

$$
q_{s}=\left(\Delta s / C_{1}\right)^{1 / \alpha},
$$

$\Delta s$ is the sampling dimension defined as:

$$
\Delta s=1+\log N_{s} / \log \lambda
$$

where $N_{s}$ is the number of independent realisations and $\lambda$ is the ratio between the largest and the smallest scales of the inertial subrange. From Eqs. (3.44) and (3.45), it comes that $q_{s}$ increases with the number of independent realisations. First- and second-order multifractal phase transitions then respectively occur when $q_{c}<q_{s}$ and $q_{c} \geq q_{s}$. In this context, it is important to mention a fundamental advantage of the log-Lévy model over the log-Poisson model. The log-Poisson 
model, as recently discussed [22], is implicitly limited by the upper bound on the intermittencies it can detect, even in case of infinite sample size. On the contrary, for the log-Lévy model, there is no theoretical upper bound for the intermittencies. This is illustrated in Fig. 8 and Table 1. The log-Lévy models capture the nonlinearity of the empirical exponents $\zeta_{S}(q)$ independently of the number of samples used to estimate $\zeta_{S}(q)$, the value of the critic order of moment $q_{s}$ (Fig. 8), and the related parameters $H, C_{1 \phi}$ and $\alpha_{\phi}$ (Table 1). In contrast, the log-Poisson fit and the values of the parameters $\gamma$ and $c$ are strongly linked to the number of samples used in the analyses (Fig. 8; Table 1).

These results have strong implications in terms of both appropriately sampling the marine environment and understanding the nature of the related biophysical patterns. The previous results demonstrate that the log-Lévy models are much more robust than the log-Poisson models to reliably quantify the intermittency properties of marine scalars through their ability to capture their statistical properties even with a limited number of observations. This is critical for the implementation of sampling strategies, and the subsequent reliable assessment of intermittent patterns and processes.

\begin{tabular}{|c|ccc|ccc|}
\hline & \multicolumn{3}{|c|}{ Temperature } & \multicolumn{3}{c|}{ Salinity } \\
\hline & $N_{1}$ & $N_{2}$ & $N_{3}$ & $N_{1}$ & $N_{2}$ & $N_{3}$ \\
\hline $\mathrm{H}$ & 0.41 & 0.42 & 0.42 & 0.43 & 0.43 & 0.42 \\
$C_{1}$ & 0.04 & 0.04 & 0.04 & 0.06 & 0.06 & 0.06 \\
$\alpha$ & 1.80 & 1.82 & 1.80 & 1.80 & 1.80 & 1.80 \\
$\gamma$ & 0.25 & 0.20 & 0.18 & 0.28 & 0.23 & 0.20 \\
$\mathrm{c}$ & 0.79 & 0.56 & 0.46 & 0.82 & 0.63 & 0.52 \\
\hline
\end{tabular}

Table 1: Parameters of the log-Lévy $\left(H, C_{1}\right.$ and $\left.\alpha\right)$ and log-Poisson $(\gamma$ and $c)$ models obtained for $500(\mathrm{~N} 1), 100(\mathrm{~N} 2)$ and $1(\mathrm{~N} 3)$ independent realisations of segments of 128 points using a temperature data set of 65,536 points. The log-Poisson parameters are strongly dependent to the number of independent realisations considered in the structure function analysis. In contrast, the log-Lévy model returns universal values for $H, C_{1}$ and $\alpha$.

\subsection{Spikes, intermittence and power spectral analysis: demixing apples and oranges}

A recent paper [72] emphasised the limitations of power spectral analysis and the fact that the spectral slope is not necessarily a strong test of any model of physical-biological interactions without a preliminary careful examination of the raw data. For instance, different phase relationships of the various sines and cosines making up the Fourier decomposition of the data may indeed lead qualitatively very distinct signals to return a $k^{-5 / 3}$ spectral slope; see [72], his Fig. 13. It is, however, widely acknowledged that a careful examination of the raw data to identify specific features (e.g. first- and second-order stationarity) that might be driving the spectral slope is an absolute 

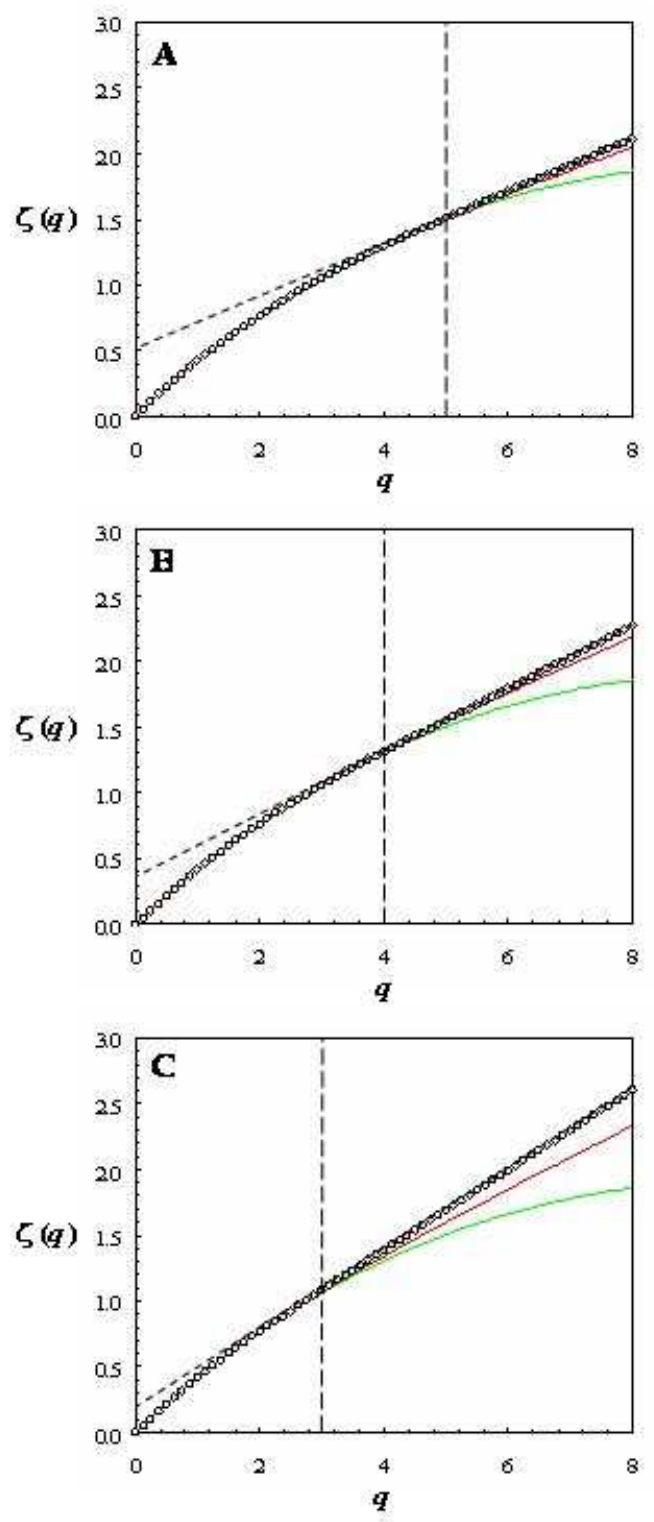

Figure 8: Empirical values of the structure function exponents $\zeta(q)$ obtained from 500 realisations $\left(\zeta_{500}(q), a\right), 100$ realisations $\left(\zeta_{100}(q)\right.$, b) and one realisation $\left(\zeta_{1}(q), \mathrm{c}\right)$ of segments of 128 points using a temperature data set of 65,536 points. As theoretically expected, the critical order of moment $q$ (above which becomes linear due to sampling limitations) decreases with decreasing independent realisations. The log-Lévy (green) and log-Poisson (red) models fit the nonlinear part of the function $\zeta(q)$ very well (i.e. for $q \leq q_{c}$ ), but the log-Lévy exhibits a universal shape whatever the number of samples taken into account. In contrast, the log-Poisson model is intrinsically dependent on the number of samples used in the analysis. The critical order of moment $q$ are indicated by the vertical dashed lines. 
prerequisite to spectral analysis [73,74], and ultimately to any type of time series analysis; see e.g. [75-77].

Franks [72] found that the power spectra of images of phytoplankton fluorescence gathered with an imaging fluorometer were 'white' $(\beta=0)$ over nearly two decades. He subsequently claimed that "the few, large, intensely fluorescent cells" that occur over a background of low fluorescence, and might somehow be related to the fluorescence intermittent hot spots clearly visible in Fig. 4d, "cause the spectrum to be flat, or white". To support this statement and his empirical findings, under the assumption that fluorescence hot spots behave as delta functions, he created 400 data series 256 points with 20 randomly placed delta functions, calculated their spectra, averaged them and plotted the average spectrum, which was flat. Similarly, he showed that randomly adding 30 delta functions of increasing amplitudes to a synthetic data set 256 points long created from 30 sine waves whose amplitude was determined by a $k^{-5 / 2}$ spectral slope leads to lower the slope of the spectrum from $k^{-5 / 2}$ with no spikes, to $k^{-2 / 3}$ when the spikes were $5 \times$ the amplitude of the largest sine wave. As stated by Franks [72], it is agreed that "spikes in plankton could arise from any number of causes that have nothing to do with mixing in 3D isotropic turbulence". However, considering that fluorescence hot spots, whether they are created by large cells, aggregates, pieces of seaweed or zooplankton guts, behave as randomly distributed delta functions is a very strong assumption. This implies that the distribution of these hot spots follow a Markov process, thus return a 'white', memoryless power spectrum, which contradicts (i) many empirical works that found spectral slopes significantly different from zero for nutrients, phyto- and zooplankton distributions [4-7,10,41,42,78-81], and (ii) more specific investigations specifically dealing with the scaling properties of intermittent behaviours in nutrient, phytoplankton and zooplankton [3-7,1012]. In addition, from a purely methodological point of view, the power spectrum resulting from randomly placed delta functions will intrinsically return a 'white' behaviour because the Fourier transform of a delta function is a constant, i.e. a white spectrum. In contrast, there is no assumption related to the use of structure functions, which would pick up the stochastic properties of any intermittent field whatever they are, i.e. flat or steep spectra.

The role of intermittent fluctuations (see Figs. 1, 3 and 5) on power spectral slopes is clarified hereafter on both theoretical and empirical grounds. From Eqs. (3.31) and (3.37), Eq. (3.5) can be rewritten as:

$$
E_{V}(k) \approx k^{-\left(5 / 3-\left(C_{1 s} /\left(\alpha_{s}-1\right)\right)\left((2 / 3)^{\alpha_{s}}-2 / 3\right)\right)} .
$$

This leads to a slope steeper than $5 / 3$ because $K_{\epsilon}(2)<0$ (see Eq. (3.37)). Using $C_{1 \epsilon}=0.15$ and $\alpha_{e}$ psilon $=1.50$ for atmospheric turbulence and $C_{1 \epsilon}=0.16$ and $\alpha_{e}$ psilon $=1.55$ for oceanic turbulence ([22]; Table 2) in Eq. (3.46) leads to $\beta_{V}(k)=1.70$. Similarly, from Eqs. (3.31) and (3.38), Eq. (3.6) is rewritten as:

$$
E_{V}(k) \approx k^{-\left(1+2 H-\left(C_{1 \phi} /\left(\alpha_{\phi}-1\right)\right)\left((2)^{\alpha} \phi-2\right)\right)} .
$$

Using $C_{1 \phi}=0.04$ and $\alpha_{\phi}=1.70$ for in vivo fluorescence advected by fully developed turbulence in the coastal waters of the eastern English Channel in Eq. (3.47) leads to $\beta_{S}(k)=1.77$. Both intermittent turbulent velocity fluctuations and intermittent in vivo fluorescence fluctuations lead to 
a spectral slope steeper than the theoretical $\beta_{S}(k)=5 / 3$ expected under non-intermittent turbulent conditions. It is stressed here that Eq. (3.47) does not involve any assumption on phytoplankton concentration being considered as a passive conservative tracer or a biologically active tracer. In contrast, Eq. (3.47) is general and would lead to a steepening of any power spectrum due to intermittency correction, whether it follows a $k^{-5 / 3}$ power law or not.

It is finally stressed that the divergences observed between Franks' conclusions [72] and those presented here might also stem from the differences in the approaches used. First, the observational platforms used by Franks and co-workers [72,82] return horizontal $32 \times 32 \mathrm{~cm}$ 2D fluorescence distributions sampled vertically every 6 to $24 \mathrm{~cm}$, significantly differ from the fluorescence data analysed here that correspond to a time series recorded from a fixed depth at a rate of $2 \mathrm{~Hz}$. Second, Franks data were collected in stratified waters 250-450 m deep $10 \mathrm{~km}$ offshore of San Diego (California, USA) where chlorophyll a concentrations were typically bounded between 0.1 and $0.6 \mu \mathrm{g}^{-1}$; [82]; their figure 2 . In contrast, the data analysed here were collected in the tidally mixed shallow coastal waters of the eastern English Channel where chlorophyll a concentrations ranged from 6 to $30 \mu \mathrm{g} \mathrm{l}^{-1}$. It is then likely that the different spectral shapes returned by the analysis of those two data sets might also be related to their intrinsic differences. Unambiguously demixing apples and oranges in an intermittent context would then request further work through a thorough investigation of data sets collected following the different methods described above simultaneously in a range of marine environments.

\section{Lagrangian turbulence and intermittency}

\subsection{Lagrangian scaling relations for velocity and passive scalars}

In a Lagrangian framework, the scaling relations given by Eqs. (3.7) and (3.8) are now a function of the time between observations instead of the spacial separation considered in the Eulerian framework. Replacing $\Delta V_{l} / l$ by $1 / t$ in Eqs. (3.7) and (3.8) leads to:

$$
\epsilon_{t} \approx \frac{\Delta V_{l}^{2}}{t}
$$

and the scalar variance flux $\chi$ as:

$$
\chi_{t} \approx \frac{\Delta S_{l}^{2}}{t}
$$

where $\Delta V_{t}=|V(\tau+t)-V(\tau)|$ and $\Delta S_{t}=|S(\tau+t)-S(\tau)|$ are the velocity shear and passive scalar gradients for an element of fluid at the scale $t$. The scalar variance flux in Eq. (4.2) does not depend anymore on a cross-product of velocity and passive scalar fluxes as in the Eulerian framework; see Eq. (3.8). The related scaling relations then

$$
\begin{gathered}
\left\langle\left(\epsilon_{t}\right)^{q}\right\rangle \approx \Lambda^{K_{\epsilon}(q)} \approx t^{-K_{\epsilon}(q)}, \\
\left\langle\left(\chi_{t}\right)^{q}\right\rangle \approx \Lambda^{K_{\chi}(q)} \approx t^{-K_{\chi}(q)},
\end{gathered}
$$




$$
\begin{aligned}
& \left\langle\left|\Delta V_{t}\right|^{q}\right\rangle \approx \Lambda^{-\zeta_{V}(q)} \approx t^{\zeta_{V}(q)}, \\
& \left\langle\left|\Delta S_{t}\right|^{q}\right\rangle \approx \Lambda^{-\zeta_{S}(q)} \approx t^{\zeta_{S}(q)},
\end{aligned}
$$

where $\Lambda=T / t$ is the scale ration between the fixed outer time-scale $T$ and the actual time-scale $t$, and the Lagrangian scaling moment functions for the velocity and passive scalar fluxes $K_{\epsilon}(q)$ and $K_{\chi}(q)$ are given by:

$$
\begin{aligned}
& K_{\epsilon}(q)=q-\zeta_{V}(2 q), \\
& K_{\chi}(q)=q-\zeta_{S}(2 q) .
\end{aligned}
$$

The fluxes are still assumed to be conservative, i.e. $K_{\epsilon}(1)=0$ and $K_{\chi}(1)=0$, which implies $\zeta_{V}(2)=1$ and $\zeta_{S}(2)=1$ [5]. As Eq. (3.31) still holds in the Lagrangian framework, it is stressed that there is no intermittency correction for the second moment, corresponding exactly to a power spectrum $E(f)=f^{-2}$. The difference in spectral slope can then be used to identify Lagrangian and Eulerian regimes in oceanic data. This is illustrated in Fig. 9 where two distinct scaling regimes are identified for temperature and salinity simultaneously recorded during 5 hours (36,000 data points) at $2 \mathrm{~Hz}$ from a drifting platform in the coastal waters of the eastern English Channel in November 1996. The sensors were located at a single depth $(10 \mathrm{~m})$ to avoid any smearing and averaging of the signals due to the length of the ship plumbing. To avoid any contamination of the signal by swell and surface waves, the sampling experiments have been conducted under weak wind $(<1$ $\mathrm{m} / \mathrm{s}$ ) conditions. The dissipative nature of the English Channel also ensures the absence of any stratification, and thus any contamination by internal gravity waves. Note that the change of scaling behaviour occurs at a frequency of $0.04 \mathrm{~Hz}$, i.e. 22 seconds. Using the mean tidal drift observed during the experiment $\left(0.55 \mathrm{~m} \mathrm{~s}^{-1}\right)$, the corresponding spatial scale is estimated as $12.3 \mathrm{~m}$, which cannot be distinguished from the size of the ship used during the sampling experiment, i.e. 12.5 $\mathrm{m}$. For time scales smaller and larger than 22 seconds, the spectral slopes of both temperature and salinity are close to the Eulerian and Lagrangian values $-5 / 3$ and -2, respectively (Fig. 9).

The Lagrangian velocity structure function scaling exponents $\zeta_{V}(q)$ and the Lagrangian passive scalar structure function scaling exponents $\zeta_{S}(q)$ are estimated from Eqs. (4.5) and (4.6), and are respectively given by:

$$
\begin{aligned}
& \zeta_{V}(q)=\frac{q}{2}-\frac{C_{1 \epsilon}}{\alpha_{\epsilon}-1}\left(\left(\frac{q}{2}\right)^{\alpha_{\epsilon}}-\frac{q}{2}\right), \\
& \zeta_{S}(q)=\frac{q}{2}-\frac{C_{1 \chi}}{\alpha_{\chi}-1}\left(\left(\frac{q}{2}\right)^{\alpha_{\chi}}-\frac{q}{2}\right),
\end{aligned}
$$

where $K_{\epsilon}(q)=\frac{C_{1 \epsilon}}{\alpha_{\epsilon}-1}\left(\left(\frac{q}{2}\right)^{\alpha_{\epsilon}}-\frac{q}{2}\right)$ and $K_{\chi}(q)=\frac{C_{1 \chi}}{\alpha_{\chi}-1}\left(\left(\frac{q}{2}\right)^{\alpha_{\chi}}-\frac{q}{2}\right) ; C_{1 \epsilon}$ and $\alpha_{\epsilon}$, and $C_{1 \chi}$ and $\alpha_{\chi}$ are the parameters of the log-Lévy model describing the velocity and passive scalar fluxes. Note that $C_{1 \epsilon}$ and $C_{1 \chi}$ can be directly estimated from Eqs. (4.9) and (4.10) as $C_{1 \epsilon}=1-2 \zeta_{V}^{\prime}(2)$ and $C_{1 \chi}=1-2 \zeta_{S}^{\prime}(2)$. The temperature structure functions estimated from Eq. (4.6) are shown in 


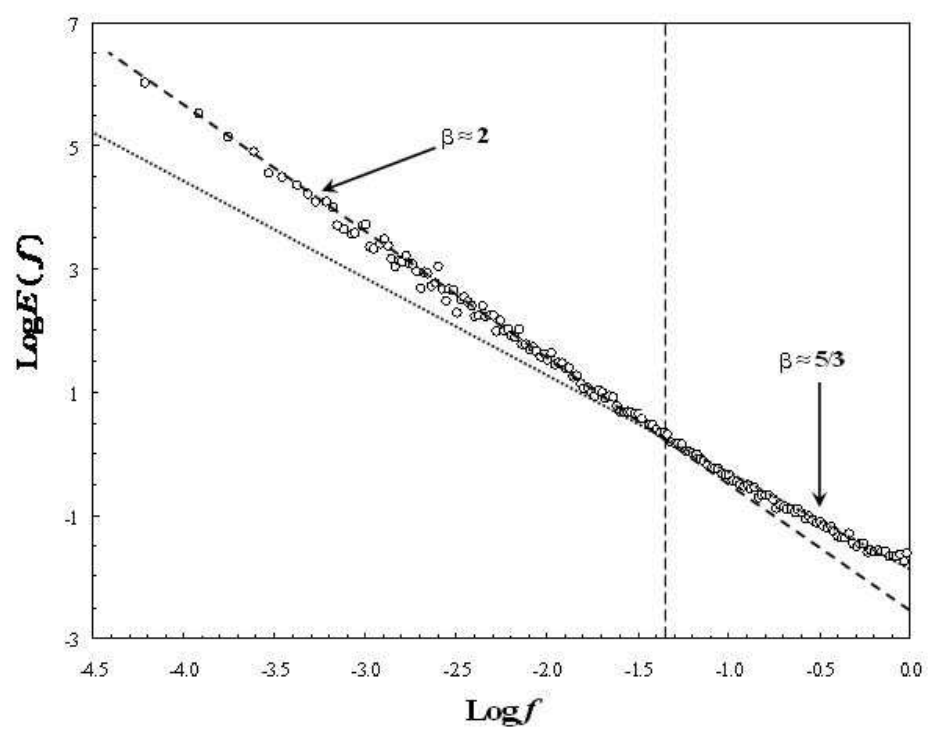

Figure 9: Log-log plot of the power spectrum of temperature fluctuations recorded in the eastern English Channel from a drifting boat $12.5 \mathrm{~m}$ long. Two scaling ranges with $E(f) \approx f^{-2}$ and $E(f) \approx f^{-5 / 3}$ respectively occur above and below a critical time-scale $t=22$ seconds. The high and low frequency regimes correspond to Eulerian and Lagrangian regimes, respectively.

Fig. 10. The transition between the Eulerian and Lagrangian regimes is very clear at $t=22$ seconds. The empirical values of $\zeta_{S}(q)$ for Eulerian and Lagrangian temperature fluctuations are nonlinear and convex, and very distinct from the non-intermittent linear cases $\zeta_{S}(q)=q H$ and $\zeta_{S}(q)=q / 2$ (Fig. 10). The parameters estimated for temperature and salinity from $\zeta_{S}(q)$ are $H=0.39, C_{1 \chi}=0.043$ and $\alpha_{\chi}=1.75$ for the Eulerian scaling range and $C_{1 \chi}=0.056$ and $\alpha_{\chi}=1.86$ for the Lagrangian scaling range.

\subsection{Scaling relations for biologically active scalars}

For chemically [83] or biologically $[84,85]$ active scalars in turbulence, there is a characteristic time, i.e. an exponential decay (e.g. first order chemical reactions) or an exponential population growth, which leads to flatten the $-5 / 3$ power law expected in the case of a purely passive scalar down to a slope of -1 (as in the case of the Batchelor [23] convective subrange; see [5]) for frequencies smaller than the corresponding characteristic frequency. For frequencies smaller than the characteristic frequency of the exponential law, the latter imposes its frequency, and the flux of variance is not ruled by turbulence anymore, because it is too slow compared to the chemical reaction or population growth. Therefore, the inverse of the local eddy turnover time $1 / t=\Delta V_{l} / l$ in Eq. (4.2) must be replaced by this characteristic frequency $\omega$, which gives:

$$
\chi_{(F)} \approx\left(\Delta F_{l}\right)^{2} \omega_{c}
$$




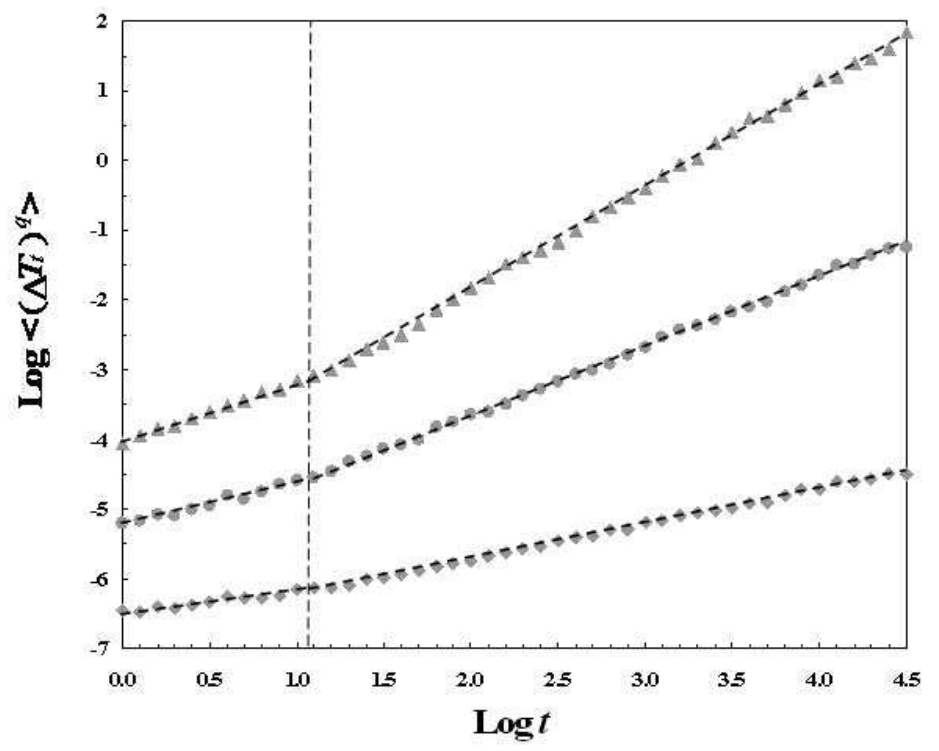

Figure 10: The temperature structure functions $\left\langle\left(T_{t}\right)^{q}>\right.$ versus $t$ in $\log$ - $\log$ plots for $q=1,2$ and 3 from bottom to top. The straight lines indicate the best power law fits over the Eulerian and Lagrangian regimes from scales smaller and greater than 22 seconds. The Lagrangian exponents corroborate the theoretical expectations $\zeta(1)=0.5$ and $\zeta(2)$.

where $F$ is the fluorescence concentration (a proxy of the phytoplankton concentration) and $\chi_{(F)}$ is still the flux of scalar variance. The scalar variance being the Fourier transform of its spectrum:

$$
\left(\Delta F_{l}\right)^{2} \approx E_{F}(k) k
$$

the power spectrum of the biologically active scalar is:

$$
E_{F}(k) \approx\left(\Delta F_{l}\right)^{2} k^{-1} \approx \chi_{(F) l} \omega_{c}^{-1} k^{-1} .
$$

From Eq. (3.31), Eq. (4.13) leads to:

$$
\zeta_{F}(2)=0 .
$$

Then, from Eq. (4.10), Eq. (4.14) implies that [15]:

$$
\zeta_{F}(q)=-K_{\chi}\left(\frac{q}{2}\right),
$$

where $K_{\chi}(q)$ is the scaling exponent of the flux of fluorescence scalar variance. Because this flux is conservative (i.e. $K_{\chi}(1)$ ), the biologically active scalar is not conservative, i.e. $\zeta_{F}(1)=$ $-K_{\chi}(1 / 2)$.

The intermittent properties of biologically active scalars have been investigated via a time series of in vivo fluorescence recorded at $2 \mathrm{~Hz}$ with a Sea Tech fluorometer adrift in the coastal waters of 

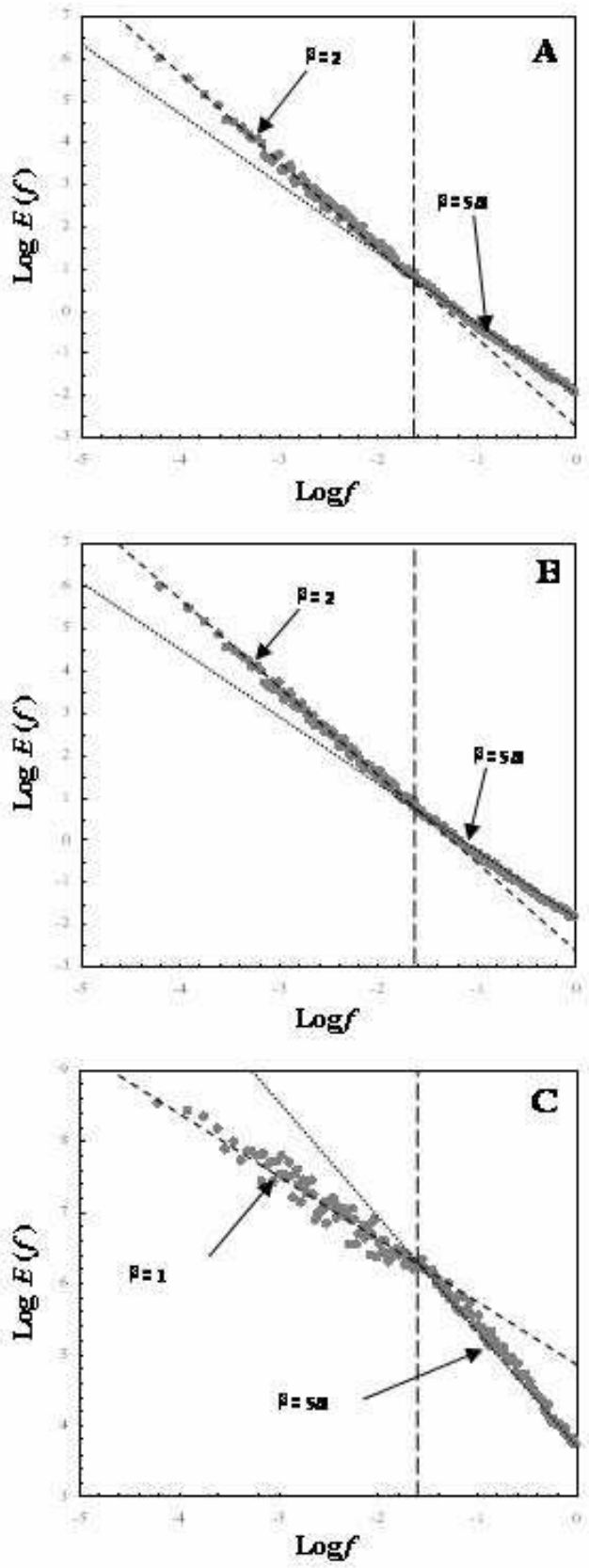

Figure 11: Log-log plots of the power spectra of temperature (a), salinity (b) and in vivo fluorescence (c) fluctuations recorded in the eastern English Channel from a drifting boat $24.9 \mathrm{~m}$ long. Two scaling ranges with $E(f) \approx f^{-2}$ and $E(f) \approx f^{-5 / 3}$ occur for scales larger and smaller than 44.7 seconds for temperature (a) and salinity (b). In contrast, the fluorescence spectrum exhibits a $E(f) \approx f^{-5 / 3}$ behaviour over the Eulerian regime, but a $E(f) \approx f^{-1}$ behaviour indicative of biological activity over the Lagrangian regime. 
the eastern English Channel aboard the N/O 'Côte de la Manche' (CNRS-INSU) on April 2, 1998. Temperature and salinity were simultaneously recorded at $2 \mathrm{~Hz}$ with a Sea-Bird 25 Sealogger CTD probe. The double logarithmic power spectra for the in vivo fluorescence, temperature and salinity time series present a mixed behaviour with two scaling regimes (Fig. 11). The change of behaviour of the power spectra occurring for a frequency $f=0.02 \mathrm{~Hz}$, which corresponds to a characteristic time scales $t=44.7$ seconds. This transition scale can be related with spatial scales using the Taylor's hypothesis of frozen turbulence [86], which basically states that temporal and spatial averages $t$ and $l$, respectively can be related by a constant velocity $V, l=V t$. Then, using the mean tidal drift observed during the field experiment $\left(v=0.55 \mathrm{~m} \mathrm{~s}^{-1}\right)$, the related length scale was 24.6 meters. This length scale is undistinguishable from the size of the ship used during the sampling experiment $(24.9 \mathrm{~m})$, which generalised the observations conducted from a smaller boat and reported in Section 4.1. For scales smaller and larger than 44.7 seconds, temperature and salinity spectra are very close from the theoretical Eulerian and Lagrangian values $-5 / 3$ and -2 , respectively (Fig. 11a, b). In contrast, fluorescence power spectrum fits well to the Eulerian $-5 / 3$ spectral slope for scales smaller than 44.7 seconds, but significantly diverges from the Lagrangian -2 slope, with $\beta_{F}=1$ over the Lagrangian scaling range (Fig. 11c).

A similar break is observed in the scaling of the structure functions of temperature, salinity and fluorescence (not shown). The resulting structure function exponents for temperature and salinity were undistinguishable and values for $C_{1 \chi}=0.045$ and $\alpha_{\chi}=1.75$ for the Eulerian scaling range and $C_{1 \chi}=0.06$ and $\alpha_{\chi}=1.85$ for the Lagrangian scaling range that were similar to those observed in Section 4.1 for temperature and salinity. Note that using the above-mentioned parameters in Eq. (4.10) leads to perfectly fits empirical data (Fig. 12a). The behaviours of the structure function exponents for in vivo fluorescence were very similar to those obtained for temperature and salinity for Eulerian scales (Fig. 12b). In contrast, they were very specific for Lagrangian scales, and satisfied the conditions $\zeta_{F}(2)=0$ (Fig. 12c). The corresponding exponents Lévy exponents were $C_{1 \chi}=0.038$ and $\alpha_{\chi}=1.84$ for the Eulerian scaling range and $C_{1 \chi}=0.034$ and $\alpha_{\chi}=1.62$ for the Lagrangian scaling range. The slight differences observed in the Eulerian $C_{1 \chi}$ and $\alpha_{\chi}$ values between fluorescence and temperature/salinity nevertheless suggest that in vivo fluorescence (i.e. phytoplankton biomass) can be considered as an active scalar diverging from the behaviours exhibited by purely passive scalars such as temperature and salinity even when the Fourier spectrum is close to the expected $-5 / 3$ (see Fig. 11). The Eulerian intermittent correction $K_{\chi}(q)$ is then different for phytoplankton biomass than for purely passive scalars. In turn, the specific spectral slope exhibited by in vivo fluorescence, is indicative a biological activity that overcomes the effects of turbulent diffusion ( $\beta_{F}=1$; Fig. 11c). More specifically, introducing $C_{1 \chi}=0.34$ and $\alpha_{\chi}=1.62$ in the function $K_{\chi}(q)$ in Eq. (4.15) leads to an extremely good fit between the theoretical and empirical functions $\zeta_{F}(q)$ up to order of moment $q=3.5$ after which the empirical function exhibited an asymptotic behaviour due to the finite size of the data set (Fig. 12c). This provides the first empirical validation of the theoretical expression $\zeta_{F}(q)$ (Eq. (4.15)) originally introduced in [5] as a generalisation of the early work of Denman and Platt [84] to describe the flattening of power spectral densities due to biological activity. 

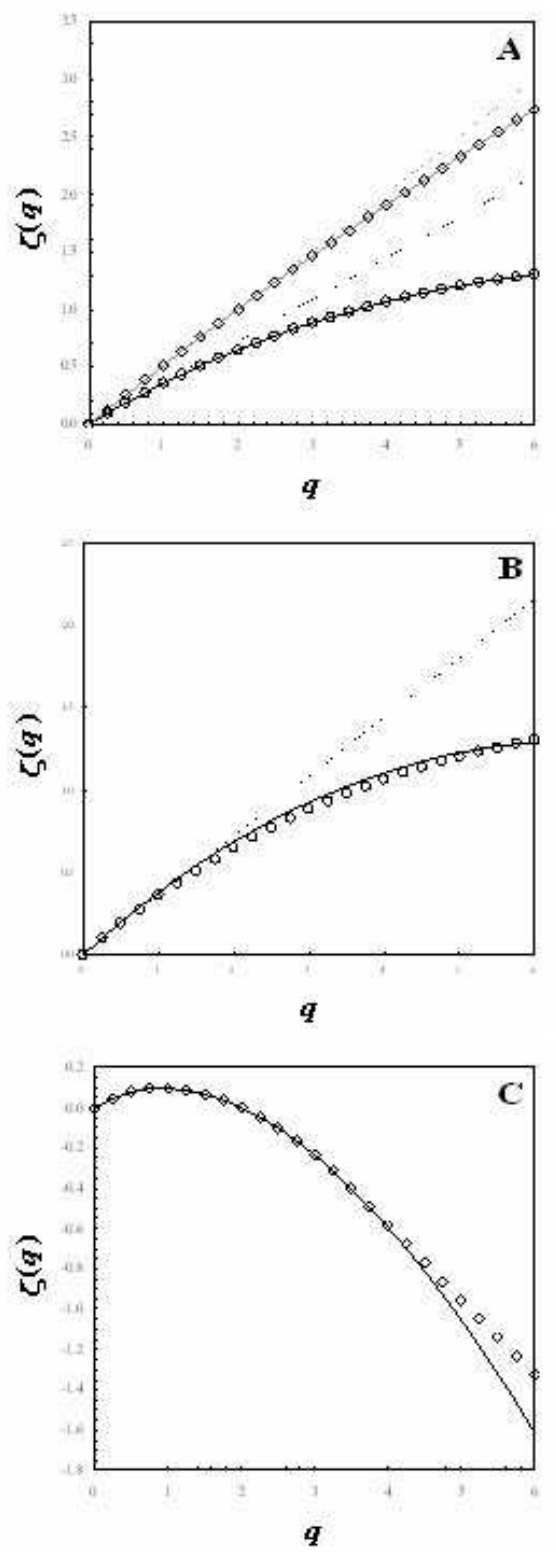

Figure 12: (a) Empirical values of the structure function exponents for salinity time series recorded in the eastern English Channel from a drifting boat $24.9 \mathrm{~m}$ long for Eulerian scales (open dots) and Lagrangian scales (open diamonds). The log-Lévy models fit the empirical data very well for Eulerian (black curve) and Lagrangian scales (grey curves). The dotted lines are the theoretical exponents $\zeta(q)=q H$ and $\zeta(q)=q / 3$ expected in case of non-intermittent Eulerian and Lagrangian turbulence, respectively. (b) Eulerian and (c) Lagrangian exponents zeta $(q)$ for in vivo fluorescence (open dots) compared to those obtained from the simultaneously recorded salinity time series. The dotted lines in (b) and the continuous black curve in (c) are the theoretical exponents $\zeta(q)=q H$ and the model $\zeta_{F}(q)=-K_{\chi}(q / 2)$ proposed to describe the intermittent fluctuation of a biologically active scalar with a $E(f) \approx f^{-1}$ spectral signature. 


\section{Turbulence intermittency: consequences for marine life}

The influence of turbulence on marine organisms is intrinsically linked to the spatial domain they belong to. Benthic and pelagic organisms will thus experience turbulence from a Eulerian and a Lagrangian perspective, respectively. On the basis of probabilistic arguments derived from the intermittent descriptions of Eulerian and Lagrangian turbulence, the next section illustrates how taking into account turbulence intermittency can lead to substantial changes in (i) the probability of occurrence of rare events, and our subsequent understanding of their impact on marine life, and (ii) salient processes in the ocean such as predator-prey encounter rates, nutrient fluxes around phytoplankton cells, physical coagulation of phytoplankton cells and in the subsequent phytoplankton aggregate volume. However, as I am not aware of any Lagrangian description of the intermittent properties of turbulent velocities so far, and considering the relative similarities between intermittent Eulerian and Lagrangian passive scalar fluctuations (see e.g. [5,87]), the following arguments will be based on previous parameterisation of Eulerian microscale turbulence.

\subsection{Intermittency, local dissipation rate and zooplankton swimming abili- ties}

Using the log-normal theory, Yamazaki et al. [88] investigated the probability density function of locally averaged dissipation rate over a sub-domain $l$ within a parent domain $L$. Considering that the minimum averaging scale (i.e. the smallest scale reachable by turbulent fluctuations) is 10 times the Kolmogorov length scale $l_{k}\left(l_{k}=\left(\nu^{3} / \epsilon\right)^{1 / 4}\right.$, where $\nu$ is the kinematic viscosity), they focused on two typical conditions in the upper ocean: turbulence in the surface mixed layer and a turbulent patch in a seasonal thermocline. In both cases, the turbulence is characterized by the average dissipation rate $\epsilon\left(10^{-5}\right.$ and $10^{-8} \mathrm{~m}^{2} . \mathrm{s}^{-3}$, respectively), and the subsequent Kolmogorov length scale $l_{k}\left(5.6210^{-4}\right.$ and $3.1610^{-3} \mathrm{~m}$, respectively), the largest external scale $L$ (10 and $1 \mathrm{~m}$, respectively) and the smallest scale $l\left(l=10 l_{k}\right)$ reachable by turbulent fluctuations $\left(5.6210^{-3}\right.$ and $3.1610^{-2} \mathrm{~m}$, respectively). Assuming log-normality for the local dissipation rate $\epsilon_{l}$, they estimated the probability for a local value $\epsilon_{\lambda}$ to exceeds the average value $\epsilon$, i.e. $\operatorname{Pr}\left(\epsilon_{l} \leq \epsilon\right.$, as 0.248 and 0.328 for the mixed layer and the seasonal thermocline case, respectively. In the log-Lévy framework, this probability is given by [6]:

$$
\operatorname{Pr}\left(\epsilon_{l} \leq \lambda^{\gamma}\right)=\lambda^{-c(\gamma)},
$$

$\gamma$ is a given singularity (i.e. an intermittency level), $\lambda$ is the scale ratio between the largest external scale $L$ and the actual scale $l$ (i.e. $\lambda=L / l$ ), and $c(\gamma)$ is the codimension function characterising the sparseness of intermittency $\gamma[6,22]$ :

$$
c(\gamma)=C_{1}\left(\frac{\gamma}{C_{1} \alpha^{\prime}}+\frac{1}{\alpha}\right)^{\alpha^{\prime}},
$$

where $C_{1}$ and $\alpha$ are the parameters characterizing the log-Lévy distribution defined above, and $\alpha^{\prime}$ is given by $1 / \alpha+1 / \alpha^{\prime}=1$ [16]. Using values of $C_{1}$ and $\alpha$ used in section 3 for turbulent velocity 
fluctuations $\left(C_{1 \epsilon}=0.16\right.$ and $\left.\alpha_{\epsilon}=1.55\right)$ and of $\lambda$ introduced, the probability $\operatorname{Pr}\left(\epsilon_{l} \geq \epsilon\right)$ became $\operatorname{Pr}\left(\epsilon_{l} \geq \epsilon\right)=0.634$ and $\operatorname{Pr}\left(\epsilon_{l} \geq \epsilon\right)=0.765$ for the mixed layer and the seasonal thermocline case, respectively.

Using root-mean-square turbulent velocity values estimated from dissipation rates observed in the seasonal thermocline and in a fjord, Yamazaki \& Squires [89] showed that nominal swimming speeds of several zooplankton species taken from a literature survey are larger than turbulent velocity fluctuations. They subsequently claimed than organism motion can be independent of the local turbulent flow field. Similar results have been recently obtained on the basis of behavioural observations of the calanoid copepod Temora longicornis [90]. It is shown that root-mean-square turbulent velocity overcomes the swimming velocity of $T$. longicornis only for very high values of the dissipation rates $\epsilon\left(\epsilon \geq 10^{-5} \mathrm{~m}^{2} \cdot \mathrm{s}^{-3}\right)$. However, these statements are based on investigations of mean values of the dissipation rate $\epsilon$, instead of the local values $\epsilon_{l}$. Using the log-Lévy framework, we will show how these results can be quantitatively refined considering the intermittent properties of $\epsilon_{l}$.

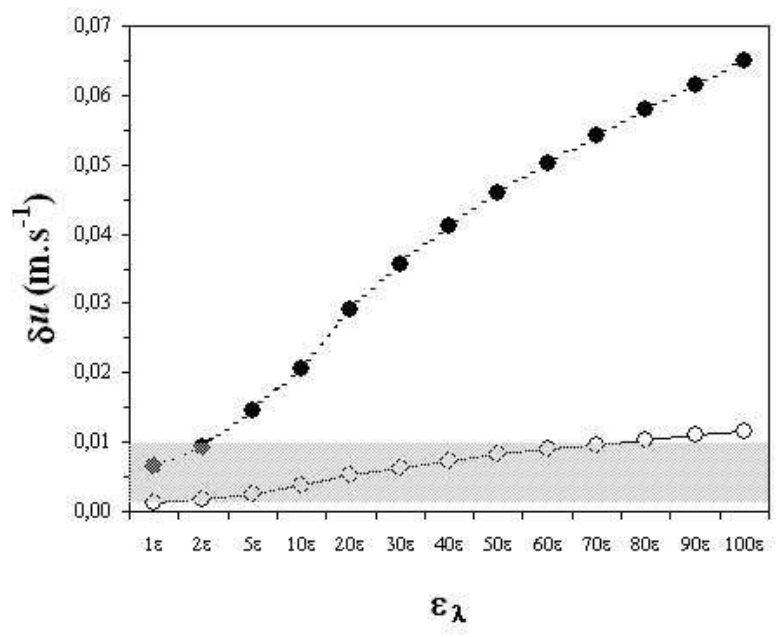

Figure 13: : Estimates of the velocity difference $l$ between two points separated by a distance of the in the surface mixed layer (black dots) and in a turbulent patch in the seasonal thermocline (open circles) for values of the local dissipation rate $\epsilon_{\lambda}$ ranging between $\epsilon$ and $100 \epsilon$. The values of the scale $l$ and of the average dissipation rate $\epsilon$ are $5.6210^{-3}$ and $3.1610^{-2} \mathrm{~m}$, and $10^{-5}$ and $10^{-8}$ $\mathrm{m}^{2} . \mathrm{s}^{-3}$ for the mixed layer and the seasonal thermocline case, respectively. The average swimming speed of most mesozooplankton species, bounded between 0.001 and $0.010 \mathrm{~m} . \mathrm{s}^{-1}$, is shown by the shaded area.

Under the isotropy assumption, the velocity difference $\partial u$ between two points separated by a distance $l$ can be estimated following the isotropic relation as:

$$
\epsilon \cong 7.5 \nu \overline{\left(\frac{\partial u}{\partial z}\right)^{2}} \approx 7.5 \nu\left(\frac{\delta u}{l}\right)^{2}
$$


where $\nu$ is the kinematic viscosity, ca. $10^{-6} \mathrm{~m}^{2} \cdot \mathrm{s}^{-1}$. From values of $l$ defined above, and values of $\epsilon_{l}$ ranging between $\epsilon$ and $100 \epsilon$, it can be seen that velocity differences $\partial u$ are of the same order of magnitude that zooplankton swimming ability (regarded as bounded between 0.001 and 0.010 $\mathrm{m} . \mathrm{s}^{-1}$ for most mesozooplankton species) for $\epsilon_{l}$ values up to $2 \epsilon$ and $70 \epsilon$ in the mixed layer and the seasonal thermocline, respectively (Fig. 13). Going back to the probabilistic arguments introduced by Eq. (5.1). The probability

$$
\operatorname{Pr}\left(\partial u<\nu_{z o o}\right)=\operatorname{Pr}\left(\epsilon_{\lambda} \geq 2 \epsilon\right)
$$

in the surface mixed layer, and

$$
\operatorname{Pr}\left(\partial u<\nu_{z o o}\right)=\operatorname{Pr}\left(\epsilon_{\lambda} \geq 70 \epsilon\right)
$$

in a turbulent patch in the seasonal thermocline. This leads to $\operatorname{Pr}\left(\partial u<\nu_{z o o}\right)=0.57$ for the mixed surface case, and $\operatorname{Pr}\left(\partial u<\nu_{z o o}\right) \rightarrow 1.00$ for the seasonal thermocline case. Thus, the surface mixed layer contains $57 \%$ of water volume whose shear can be overcome by mesozooplankton swimming. On the opposite, mesozooplankton swimming ability overcomes turbulent shear in almost $100 \%$ of the turbulent patches in the seasonal thermocline.

These simple arguments provide the first quantitative description of mesozooplankton swimming ability against turbulence taking into account the probabilistic nature of intermittent turbulence. These results also confirm that organism motion can be independent on the local flow field in most areas of the world ocean.

\subsection{Intermittency, local dissipation rate and matter fluxes in marine ecosys- tems}

The influence on turbulence intermittency on plankton processes will be considered through three major processes controlled by turbulent processes, and at the core of marine system functioning: nutrient fluxes around non-motile phytoplankton cells, physical coagulation of phytoplankton cells and predator-prey encounter rates.

In order to take into account the distribution of the local dissipation rate $\epsilon_{\lambda}$ in calculations, Eq. (3.10) has been rewritten as [46]:

$$
\left\langle\epsilon_{l}^{q}\right\rangle=\epsilon^{q} \lambda^{K(q)}
$$

and used Eq. (5.6) to evaluate the average of any polynomial function $f\left(\epsilon_{l}\right)$ of the intermittent field $\epsilon_{l}$ as:

$$
f\left(\epsilon_{l}\right)=\sum_{q=0}^{N} a_{q}\left(\epsilon_{l}\right)^{q},
$$

where $a_{q}$ are constants, and $q$ the polynomial order of the function $f\left(\epsilon_{l}\right)$. The average of the function $f\left(\epsilon_{l}\right)$ in Eq. (5.7) leads to: 


$$
f\left(\epsilon_{\lambda}\right)=\sum_{q=0}^{N} a_{q}(\epsilon)^{q} \lambda^{K(q)} .
$$

\subsubsection{Intermittent turbulence and nutrient fluxes around phytoplankton cells}

Following Karp-Boss et al. [91], the increased rate of nutrient flux due to turbulence around nonmotile phytoplankton cells of radius $r(\mathrm{~m})$ can be directly estimated using the Sherwood number, $S$, as:

$$
S_{1}=1+0.29\left(\frac{r}{D}\left(\frac{\epsilon_{0}}{\nu}\right)^{1 / 2}\right)^{1 / 2}
$$

for $r<l_{B}$, and

$$
S_{2}=0.55\left(\frac{r}{D}\left(\frac{\epsilon_{0}}{\nu}\right)^{1 / 2}\right)^{1 / 3}
$$

for $r>l_{B} . \quad D$ is the diffusivity $\left(D=10^{-9} \mathrm{~m}^{2} \mathrm{~s}^{-1}\right)$, the $\epsilon$ mean turbulent energy dissipation rate $\left(\mathrm{m}^{2} \mathrm{~s}^{-3}\right), \nu$ the kinematic viscosity $\left(\nu=10^{-6} \mathrm{~m}^{2} \mathrm{~s}^{-1}\right)$ and $l_{B}$ the Batchelor microscale, the scale of the smallest variations in the ambient nutrient field. One may note here that the Batchelor microscale $l_{B}$ is smaller than the Kolmogorov microscale $l_{K}$ (i.e. the scale of the smallest turbulent eddies) following $l_{K}=l_{B}(D / \nu)^{1 / 2}$.

Now let the turbulent kinetic energy dissipation rate $\epsilon$ be a intermittent variable $\epsilon_{l}$ characterized by the scaling moment function $K_{\epsilon}$ defined above, and by its mean $\epsilon_{0}=\left\langle\epsilon_{l}\right\rangle$. Consider the average Sherwood numbers $\bar{S}_{1}$ and $\bar{S}_{2}$ associated to the intermittent (multifractal) variable $\epsilon_{\lambda}$ and defined as $\bar{S}_{1}=\left\langle S_{1}\left(\epsilon_{l}\right)\right\rangle$ and $\bar{S}_{2}=\left\langle S_{2}\left(\epsilon_{l}\right)\right\rangle$, when $r<l_{B}$ and $r>l_{B}$, respectively. Using Eq. (5.8), Eqs. (5.9) and (5.10) can be rewritten as:

$$
\bar{S}_{1}=1+0.29 k_{1} \epsilon_{0}^{1 / 4} \lambda^{K_{\epsilon}(1 / 4)}
$$

for $r<l_{B}$, and

$$
\bar{S}_{2}=0.55 k_{2} \epsilon_{0}^{1 / 6} \lambda^{K_{\epsilon}(1 / 6)}
$$

for $r>l_{B}$, where $k_{1}$ and $k_{2}$ are constants. Because $\lambda \gg 1, K(1 / 4)<0$ and $K(1 / 6)<0$, therefore $\lambda^{K_{\epsilon}(1 / 4)}$ and $\lambda^{K_{\epsilon}(1 / 6)}$ act as dampening factors in Eqs. (5.11) and (5.12) yielding $\left(\bar{S}_{1} / S_{1}\right)<1$ and $\left(\bar{S}_{2} / S_{2}\right)<1$. This shows that using a mean value of the turbulent kinetic energy dissipation rate $\epsilon_{0}$ instead of the intermittent distribution $\epsilon_{l}$ leads to overestimate the turbulence contribution to the rates of nutrient fluxes around phytoplankton cells, whatever their size may be.

In order to quantify this difference, one needs to estimate the intermittent dampening factors $\left(\bar{S}_{1} / S_{1}\right)$ and $\left(\bar{S}_{2} / S_{2}\right)$ due to $\lambda^{K_{\epsilon}(1 / 4)}$ and $\lambda^{K_{\epsilon}(1 / 6)}$ in Eq. (5.11) and (5.12) using realistic values of $\lambda, K_{\epsilon}(1 / 4)$ and $K_{\epsilon}(1 / 6)$. The inertial subrange scale ratio $\lambda$ is increasing with increasing intensities of turbulence, and can be reasonably regarded as ranging between $10^{2}$ and $10^{5}$, e.g. [92]. 
The scale-invariant moment exponents $K_{\epsilon}(1 / 4)$ and $K_{\epsilon}(1 / 6)$ have been estimated from high resolution shear vertical profiles recorded in tidally mixed coastal waters following Seuront et al. [6] as $K_{\epsilon}(1 / 4)=-0.053 \pm 0.005$ and $K_{\epsilon}(1 / 6)=-0.042 \pm 0.004$ (Seuront \& Yamazaki, unpublished data). The resulting dampening factors $\left(\bar{S}_{1} / S_{1}\right)$ and $\left(\bar{S}_{2} / S_{2}\right)$ ranges between 1.06 and 1.19 , and between 1.21 and 1.62 when $r<l_{B}$ and $r>l_{B}$, respectively. This leads to consider a decrease in the rate of nutrient fluxes around phytoplankton cells due to microscale turbulence intermittency ranging between $6.26 \%$ and $19.07 \%$ for phytoplankton cells smaller than the Batchelor microscale $l_{B}$, and between $21.22 \%$ and $61.78 \%$ for phytoplankton cells larger than the Batchelor microscale $l_{B}$. The inertial subrange scale ratio $\lambda$ increases with increasing intensities of turbulence. The decrease in the rate of nutrient fluxes around phytoplankton cells of any size is thus higher when the intensity of turbulence is high.

\subsubsection{Intermittent turbulence and physical coagulation of phytoplankton cells}

Theoretical analyses of particle coagulation processes predict that aggregate formation depends on the probability of particle collision and on the efficiency with which two particles that collide and stick together afterwards $[93,94]$. The former is a function of particle concentration, size and the mechanism by which particles are brought into contact, e.g. Brownian motion, shear or the differential settlement of particles. The latter which is not studied in the present paper depends mainly on the physico-chemical properties of the particle surface and may vary with the particle type.

Let consider a monospecific phytoplankton cells suspension characterized by a cell radius $r$ (m) and cell concentration $C$ (cells.m ${ }^{-3}$ ). Because all particles are of the same size and density and settle with the same velocity, and because encounters due to Brownian motion is insignificant for particles $>1 \mu \mathrm{m}$ [93], the only mechanism that may increase the relative velocity between phytoplankton cells and thus to bring them to collide is due to turbulent shear and can be expressed as [94]:

$$
E_{1}=10.4 r^{3} C^{2}\left(\epsilon_{0} / \nu\right)^{1 / 2},
$$

where $E_{1}$ is the encounter rate due to turbulence (encounter.s ${ }^{-1}$ ), $\epsilon_{0}$ the mean turbulent energy dissipation rate $\left(\mathrm{m}^{2} \mathrm{~s}^{-3}\right)$ and $\nu$ the kinematic viscosity $\left(\nu=10^{-6} \mathrm{~m}^{2} \mathrm{~s}^{-1}\right)$. As previously done in Section 5.2.1, let the turbulent kinetic energy dissipation rate be a intermittent variable $\epsilon_{l}$ characterized by the scaling moment function $K_{\epsilon}(q)$ defined above, and by its mean $\left.\left.\epsilon_{0}=\right\rangle \epsilon_{l}\right\rangle$. Here, $E_{1}$ and $\bar{E}_{1}$ are regarded as estimates of average encounter rates, i.e. $E_{1}=E\left(\epsilon_{0}\right)$ and $\bar{E}_{1}=\left\langle E\left(\epsilon_{l}\right)\right\rangle$ when the turbulent energy dissipation rates are regarded as homogeneous and intermittent variables, respectively. In this case, Eq. (5.13) is rewritten as:

$$
\bar{E}_{1}=10.4 r^{3} C^{2}\left(\epsilon_{0} / \nu\right)^{1 / 2} \lambda^{K_{\epsilon}(1 / 2)} .
$$

This finally yields:

$$
\bar{E}_{1}=E_{1} \lambda^{K_{\epsilon}(1 / 2)}
$$


and as defined above, $\lambda \gg 1$ and $K_{\epsilon}(1 / 2)<0, \lambda^{K(1 / 2)}$ thus acts as a restraining factor therefore $\bar{E}_{1}<E_{1}$. Using the values of $\lambda$ proposed in Section 5.2.1 (i.e. $\lambda \in\left[10^{2}, 10^{5}\right]$ ) and $K_{\epsilon}(1 / 2)=$ $-0.063 \pm 0.005$ (Seuront and Yamazaki, unpublished data), the factor $\lambda_{K_{\epsilon}(1 / 2)}$ ranges between 0.48 and 0.75 . Now, the intermittent nature of microscale turbulence leads to consider a decrease in its contribution to the physical coagulation of phytoplankton cells ranging between $25 \%$ and $48 \%$. This decrease is higher when the turbulence levels are high, i.e. the inertial subrange scale ratio is large.

Considering the role played by large particle aggregates in the vertical flux of organic matter in the ocean $[95,96]$, the effects of this decrease in encounters due to intermittency on the growth in particle size is considered hereafter. It has been shown that the average solid volume of aggregates increases according to the following formula [97]:

$$
V_{t}=V_{0} e^{\alpha\left(7.8 \phi\left(\epsilon_{0} / \nu\right)^{1 / 2} / \pi\right) t},
$$

where $V_{0}$ and $V_{t}$ are the average volume of aggregates at time 0 and $t, \alpha$ is the stickiness coefficient (i.e. $\alpha \in[0,1]), \phi$ is the volume-concentration of cells $\left(\phi=(4 / 3) \pi r^{3} C_{0}\right)$. Introducing the precise statistical distributions of turbulent dissipation rate $\epsilon_{\lambda}$ in Eq. (5.16) instead of its average value $\epsilon_{0}$ leads to a decrease in the aggregate average volumes ranging between $22 \%$ and $41 \%$ in low and high hydrodynamic conditions, respectively.

\subsubsection{Intermittent turbulence and predator-prey encounter rates}

Following the seminal theory of Rothschild \& Osborn [98], Kirboe \& Saiz [99] demonstrated that the encounter rate $E$ (encounters $\mathrm{s}^{-1}$ ) between plankton predators and preys can be expressed as the sum of the encounter rate due to organism behavior and the encounter rate due to microscale turbulence. The former is a function of particle concentration, swimming speed of predator and prey and perceptive distance of the predator. The latter which I explore here in more details is expressed as:

$$
E_{2}=C \pi R^{2} w
$$

where $E_{2}$ is the encounter rate due to microscale turbulence, $C$ is the number of preys per unit volume (preys. $\left.\mathrm{m}^{-3}\right), R$ is the perceptive distance of the predator $(\mathrm{m})$, and $w\left(\mathrm{~m} \mathrm{~s}^{-1}\right)$ is the rootmean-square turbulent velocity enhancing the relative motion between predator and prey. The rms turbulent velocity $w$ is directly related to the intensity of turbulence, characterized by a mean value of the turbulent kinetic energy dissipation rate $\epsilon_{0}\left(\mathrm{~m}^{2} \mathrm{~s}^{-3}\right)$ following [98]:

$$
w=1.9\left(\epsilon_{0} d\right)^{1 / 3},
$$

where $d$ is the separation distance between predator and prey when encounter takes place, i.e. $d=R$, e.g. [100]. Finally, inserting the expression for the rms turbulent velocity $w$ [Eq. (5.18)] in Eq. (5.17) yields:

$$
E_{2}=1.9 C \pi R^{7 / 3} \epsilon_{0}^{1 / 3}
$$


Let now $\epsilon_{l}$ be a intermittent variable characterized by the scaling moment function $K_{\epsilon}(q)$ defined above, and by its mean $\left\langle\epsilon_{l}\right\rangle=\epsilon_{0}$. Here, $E_{2}$ and $\bar{E}_{2}$ are the estimates of average encounter rates due to turbulence, i.e. $E_{2}=E\left(\epsilon_{0}\right)$ and $\bar{E}_{2}=\left\langle E\left(\epsilon_{0}\right)\right\rangle$ when the turbulent energy dissipation rates are regarded as homogeneous and intermittent (multifractal) variables, respectively. Eq. (5.19) can thus be rewritten as:

$$
E_{2}=1.9 C \pi R^{7 / 3} \epsilon_{0}^{1 / 3} \lambda^{K_{\epsilon}(1 / 3)} .
$$

Now $\bar{E}_{2}=E_{2} \lambda^{K_{\epsilon}(1 / 3)}$, and as defined above, $\lambda \gg 1$ and $K_{\epsilon}(1 / 3)<0, \lambda^{K_{\epsilon}(1 / 3)}$ acts as a restraining factor therefore $\bar{E}_{2}<E_{2}$. Considering values of the inertial subrange scale ratio $\lambda$ bounded between $10^{2}$ and $10^{5}$, and $K(1 / 3)=-0.060 \pm 0.003$ (Seuront \& Yamazaki, unpublished data), the factor $\lambda^{K_{\epsilon}(1 / 3)}$ ranges between 0.50 and 0.76 . Taking into account the intermittent structure of turbulent kinetic energy dissipation rate $\epsilon_{l}$ instead of an average value $\epsilon_{0}$ then decreases the contribution of microscale turbulence to the predator-prey encounter rate of $25 \%$ to $50 \%$ for values ranging from $10^{-10}$ to $10^{-2} \mathrm{~m}^{2} \mathrm{~s}^{-3}$.

\section{Conclusions}

Historically, intermittency has seldom been described in ocean sciences. In physical oceanography, intermittency has essentially been related of its impacts on sampling, data processing, and statistical analysis [1,32], and is not referred to in specialized monographs [26-30]. In biological oceanography, the description of intermittency and the understanding of its links with patchiness and its consequences for the ecology of marine organisms have still been very poorly considered. While widely understated in ocean sciences, the intermittent behaviour of both turbulent velocity and passive scalar fluctuations is a ubiquitous property of the marine environment. As such, it is hoped that this contribution has clarified some intriguing aspects of microscale turbulence, but also opened perspectives concerning on assessing the role of intermittency on key processes such as nutrient fluxes, the formation of biological aggregates and predator-prey encounter rates. The cascade models described here can be used to faithfully represent the biological effect of turbulent processes as is found in marine systems. This precision might prove to be a fundamental requirement for integrated physical and biological models in order for their results to be meaningful.

\section{Acknowledgements}

This work has been financially supported by Flinders University, Marine Innovation South Australia, the Australian Research Council, the Centre National de la Recherche Scientifique and the University of Sciences and Technologies of Lille. 


\section{References}

[1] [1] M. A. Baker, C. H. Gibson. Sampling turbulence in the stratified ocean: statistical consequences of strong intermittency. J. Phys. Oceanogr., 17 (1987), 1817-1836.

[2] Gibson. Kolmogorov similarity hypotheses for scalar fields: sampling intermittent turbulent mixing in the ocean and galaxy. Proc. R. Soc. Lond. A, 434 (1991), 149-164.

[3] M. Pascual, F. Ascioti, H. Caswell. Intermittency in the plankton: a multifractal analysis of zooplankton biomass variability. J. Plankton Res., 17 (1995), 1209-1232.

[4] L. Seuront, F. Schmitt, Y. Lagadeuc, D. Schertzer, S. Lovejoy, S. Frontier. Multifractal structure of phytoplankton biomass and temperature in the ocean. Geophys. Res. Lett., 23 (1996), 3591-3594

[5] L. Seuront, F. Schmitt, D. Schertzer, Y. Lagadeuc, S. Lovejoy. Multifractal intermittency of Eulerian and Lagrangian turbulence of ocean temperature and plankton fields. Nonlin. Proc. Geophys., 3 (1996), 236-246.

[6] L. Seuront, F. Schmitt, Y. Lagadeuc, D. Schertzer, S. Lovejoy. Multifractal analysis as a tool to characterize multiscale inhomogeneous patterns. Example of phytoplankton distribution in turbulent coastal waters. J. Plankton Res., 21 (1999), 877-922.

[7] L. Seuront, V. Gentilhomme, Y. Lagadeuc. Small-scale nutrient patches in tidally mixed coastal waters. Mar. Ecol. Prog. Ser., 232 (2002), 29-44.

[8] D. Jou. Intermittent turbulence: a short introduction. Sci. Mar., 61 (1997), 57-62.

[9] J. Jimanez. Intermittency and cascades. Sci. Mar., 409 (2000), 99-120.

[10] S. Lovejoy, W. J. S. Currie, Y. Tessier, M. R. Claereboudt, M. R. Bourget, E. Roff, D. Schertzer. Universal multifractals and ocean patchiness: phytoplankton, physical fields and coastal heterogeneity. J. Plankton Res., 23 (2001), 117-141.

[11] L. Seuront. Hydrodynamical and tidal controls of small-scale phytoplankton patchiness. Mar. Ecol. Prog. Ser., 302 (2005), 93-101.

[12] L. Seuront, Y. Lagadeuc. Multiscale patchiness of the calanoid copepod Temora longicornis in a turbulent coastal sea. J. Plankton Res., 23 (2001), 1137-1145.

[13] L. Seuront, F. G. Schmitt, M. C. Brewer, J. R. Strickler, S. Souissi. From random walk to multifractal random walk in zooplankton swimming behavior. Zool. Stud., 43 (2004), 8-19.

[14] L. Seuront, S. C. Leterme. Increased zooplankton behavioural stress in response to short-term exposure to hydrocarbon contamination. The Open Oceanography Journal, 1 (2007), 1-7. 
[15] L. Seuront, A. C. Duponchel, C. Chapperon. Heavy-tailed distributions in the intermittent motion behaviour of the intertidal gastropod Littorina littorea. Physica A, 385 (2007), 573 582.

[16] A. M. Obukhov. Some specific features of atmospheric turbulence. J. Fluid Mech., 13 (1962), 77-81.

[17] A. N. Kolmogorov. A refinement of previous hypotheses concerning the local structure of turbulence in a viscous incompressible fluid at high Reynolds number. J. Fluid Mech., 13 (1962), 82-85.

[18] B. B. Mandelbrot. Intermittent turbulence in self-similar cascades: divergence of high moments and dimension of the carrier. J. Fluid Mech., 62 (1974), 305-330.

[19] B. B. Mandelbrot. Intermittent turbulence and fractal dimension: kurtosis and the spectral exponent 5/3 + . In: R. Teman (ed.), Turbulence and Naviers Stokes equations. Lectures notes in Mathematics, vol. 55, 121-145. Springer, 1976.

[20] H. Yamazaki. Breakage models: lognormality and intermittency. J. Fluid Mech., 79 (1990), 159-165.

[21] U. Frisch. Turbulence. Cambridge University Press, 1995.

[22] L. Seuront, H. Yamazaki, F. Schmitt. Intermittency. In: H. Baumert, J. Sundermann, J. Simpson (eds.), Marine Turbulences: Theories, Observations and Models. Cambridge University Press, Cambridge, 2005, 66-78.

[23] G. K. Batchelor. Small-scale variation of convected quantities like temperature in turbulent fluid, Part I. General discussion and the case of small conductivity, J. Fluid Mech., 1959, No. $5,113$.

[24] R. W. Stewart. Turbulence. Cambridge University Press, Cambridge, 1969.

[25] H. Baumert, J. Sndermann, J. Simpson (eds.). Marine Turbulences: Theories, Observations and Models. Cambridge University Press, Cambridge, 2005.

[26] S. Pond, G.L. Pickard. Introductory dynamical oceanography. Butterworth-Heineman, Oxford, 1983.

[27] C. P. Summerhayes, S. A. Thorpe. Oceanography. An illustrated guide. Manson Publishing, London, 1996.

[28] T. Bohr, M. H. Jensen, G. Paladin, A. Vulpiani. Dynamical systems approach to turbulence. Cambridge University Press, 1998.

[29] L. H. Kantha, C. A. Clayson. Small scale processes in geophysical fluid flows. International geophysics series, Vol. 67, 888 p., 2000. 
[30] S. B. Pope. Turbulent Flows. Cambridge University Press, Cambridge, 2000.

[31] J. Jimanez. Oceanic turbulence at millimeter scales. Sci. Mar., 61 (1997), 47-56.

[32] M. Bohle-Carbonel. Pitfalls in sampling, comments on reliability and suggestions for simulation. Cont. Shelf Res., 12 (1992), 3-24.

[33] L. Seuront, Y. Lagadeuc. Towards a terminological consensus in ecology: variability, inhomogeneity and heterogeneity. J. Biol. Syst., 9 (2001), 81-87.

[34] E. Haeckel. Plakton studien. J. Zeitschriftfuer Naturwis., 25 (1891), 232-336.

[35] A. C. Hardy. Observation of the uneven distribution of oceanic plankton. Discovery Report, 1936, No. 11, 511-538.

[36] R. M. Cassie. An experimental study of factors inducing aggregation in marine plankton. New Zealand J. Sci., 2 (1959), 339-365.

[37] D. H. Cushing. Patchiness. Rapp. Cons. Int. Explor. Mer, 153 (1962), 152-163.

[38] R. M. Cassie. Microdistribution in the plankton. Oceanogr. Mar. Biol. Ann. Rev., 1 (1963), 223-252.

[39] J. G.Mitchell, J. A. Furhman. Centimeter scale vertical heterogeneity in bacteria and chlorophyll a. Mar. Ecol. Prog. Ser., 54 (1989), 141-148.

[40] P. K. Bjrnsen, T. G.Nielsen. Decimeter scale heterogeneity in the plankton during a pycnocline bloom of Gyrodinuim aureolum, Mar. Ecol. Prog. Ser., 73 (1991), 263-267.

[41] L. Seuront, Y. Lagadeuc. Characterisation of space-time variability in stratified and mixed coastal waters (Baie des Chaleurs, Quebec, Canada): application of fractal theory. Mar. Ecol. Prog. Ser., 159 (1997), 81-95.

[42] L. Seuront, Y. Lagadeuc. Spatio-temporal structure of tidally mixed coastal waters: variability and heterogeneity. J. Plankton Res., 20 (1998), 1387-1401.

[43] R. L. Waters, J. G. Mitchell. Centimeter-scale spatial structure of estuarine in vivo fluorescence profiles. Mar. Ecol. Prog. Ser., 237 (2002), 51-63.

[44] R. L. Waters, J. G. Mitchell, J. R. Seymour. Geostatistical characterization of centimetre-scale spatial structure of in vivo fluorescence. Mar. Ecol. Prog. Ser., 251 (2003), 49-58.

[45] M. Estrada, E. Berdalet. Phytoplankton in a turbulent world. Sci. Mar., 61 (1997), 125-140.

[46] L. Seuront, F.G. Schmitt, Y. Lagadeuc. Turbulence intermittency, small-scale phytoplankton patchiness and encounter rates in plankton: where do we go from here? Deep-Sea Res. I, 48 (2001), 1199-1215. 
[47] H. Svendsen. Physical oceanography and marine ecosystems: some illustrative examples. Sci. Mar., 61 (1997), 93-108.

[48] L. P. Sanford. Turbulent mixing in experimental ecosystem studies. Mar. Ecol. Prog. Ser., 161 (1997), 265-293.

[49] G. K. Batchelor, A. A. Towsend. The nature of turbulent motion at large wavenumbers. Proc. R. Soc. A, 199 (1949), 238-250.

[50] D. C. Wilcox. Turbulence modeling for CFD. DCW Industries, 1998.

[51] A. N. Kolmogorov. The local structure of turbulence in incompressible viscous fluid for very large Reynolds numbers. Dokl. Akad. Nauk SSSR, 30 (1941), 299-303.

[52] A. S. Monin, A. M. Yaglom. Statistical Fluid Mechanics: Mechanics of Turbulence. MIT Press, 1975.

[53] A. M. Obukhov. Spectral energy distribution in a turbulent flow. Dokl. Akad. Nauk. SSSR, 32 (1941), 22-24.

[54] A. M. Obukhov. Structure of the temperature field in a turbulent flow. Izv. Akad. Nauk SSSR., Geogr. I Jeofiz., 1949, No. 13, 55.

[55] S. Corrsin. On the spectrum of isotropic temperature in an isotropic turbulence. J. Appl. Phys., 22 (1951), 469-473.

[56] A. S. Gurvich. Experimental research on frequency spectra of atmospheric turbulence. Izv. Akad. Nauk SSSR, geofiz. ser., 1960, 1042-1055.

[57] H. Yamazaki, J.G. Mitchell, L. Seuront, F. Wolk, H. Li. Phytoplankton microsctructure in fully developed oceanic turbulence. Geophys. Res. Lett., 33 (2006), L01603, doi:10.1029/2005GL024103.

[58] W. J. S. Currie, J.C. Roff. Plankton are not passive tracers: plankton in a turbulent environment. J. Geophys. Res., 111 (2006), C05S07, doi:10.1029/2005JC002967.

[59] L. Seuront, F. G. Schmitt. Multiscaling statistical procedures for the exploration of biophysical couplings in intermittent turbulence. Part I. Theory. Deep-Sea Res. II, 52 (2005), 13081324.

[60] A. M. Yaglom. Local structure of the temperature field in a turbulent flow. Dokl. Akad. Nauk SSSR, 69 (1949), 743.

[61] L. F. Richardson. Weather prediction by numerical processes. Cambridge University Press, Cambridge, 1922.

[62] A. M. Yaglom. The influence of fluctuations in energy dissipation on the shape of turbulent characteristics in the inertial interval. Sov. Phys. Dokl., 11 (1966), 26-29. 
[63] A. S. Gurvich, A. M. Yaglom. Breakdown of eddies and probability distributions for smallscale turbulence. Phys. Fluids, 10 (1967), 59-65.

[64] G. Paladin, A. Vulpiani. Anomalous scaling laws in multifractal objects. Phys. Rep., 156 (1987), 147-225.

[65] C. Meneveau, K. R. Sreenivasan. Simple multifractal cascade model for fully developed turbulence. Phys. Rev. Lett., 59 (1991), 1424-1427.

[66] W. Feller. An introduction to probability theory and its applications, Vol. II. Wiley, 1971.

[67] D. Schertzer, S. Lovejoy. Physical modeling and analysis of rain and clouds by anisotropic scaling multiplicative processes. J. Geophys. Res., 92 (1987), 96-99.

[68] S. Kida. Log-stable distribution and intermittency of turbulence. J. Phys. Soc. Japan, 60 (1991), 5-8.

[69] M.F. Shlesinger, G.M. Zaslavsky, U. Frish (Eds.), Lvy Flights and Related Topics in Physics, Springer, Berlin, 1996

[70] B. Dubrulle. Intermittency in fully developed turbulence: log-Poisson statistics and generalized scale covariance. Phys. Rev. Lett., 73 (1994), 959-962.

[71] Z. S. She, E. C. Waymire. Quantized energy cascade and log-Poisson statistics in fully developed turbulence. Phys. Rev. Lett., 74 (1995), 262-265.

[72] B. Castaing, B. Dubrulle. Fully developed turbulence: a unifying point of view. J. Phys. II France, (1995), No. 5, 895-899.

[73] B. Dubrulle. Anomalous scaling and generic structure function in turbulence. J. Phys. II France, 2006, No. 6, 1825-1840.

[74] P. J. S. Franks. Plankton patchiness, turbulent transport and spatial spectra. Mar. Ecol. Prog. Ser., 294 (2005), 295-309.

[75] M. B. Priestley. Spectral analysis and time series. Academic Press, 1983.

[76] P. Bloomfield. Fourier analysis of time series: an introduction. Wiley Interscience, 2000.

[77] C. Chatfield. The analysis of time series: an introduction. Chapman \& Hall/CRC, 2003.

[78] H. Kantz, T. Schreiber. Nonlinear time series analysis. Cambridge University Press, 2004.

[79] W. S. Wei. Time series analysis: univariate and multivariate methods. Addison Wesley, 2005.

[80] A. Tsuda, H. Sugisaki, T. Ishimaru, T. Saino, T. Sato. White-noise-like distribution of the oceanic copepod Neocalanus cristatus in the subarctic North Pacific. Mar. Ecol. Prog. Ser., (97) 1993, 39-46. 
[81] D. G. Mountain, M. H. Taylor. Fluorescence structure in the region of the tidal mixing front on the southern flank of Georges Bank. Deep-Sea Res II, 43 (1996), 1831-1853.

[82] P. H. Wiebe, D. G. Mountain, T. K. Stanton, C. H. Greene, G. Lough, S. Kaartvedt, J. Dawson, N. Copley. Acoustical study of the spatial distribution of plankton on Georges Bank and the relationship between volume backscattering strength and the taxonomic composition of the plankton. Deep-Sea Res II, 43 (1996), 1971-2001.

[83] A. J. Pershing, P. H. Wiebe, J. P. Manning, N. J. Copley. Evidence for vertical circulation cells in the well-mixed area of Georges Bank and their biological implications. Deep-Sea Res. II, 48 (2001), 283-310.

[84] P. J. S. Franks, J. S. Jaffe. Microscale variability in the distributions of large fluorescent particles observed in situ with a planar laser imaging fluorometer. J. Mar. Syst., 69 (2008), 254-270.

[85] S. Corrsin. The reactant concentration spectrum in turbulent mixing with a first-order reaction. J. Fluid Mech., 11 (1961), 407-416.

[86] K. L. Denman, T. Platt. The variance spectrum of phytoplankton in a turbulent ocean. J. Mar. Res., 34 (1976), 593-601.

[87] K. L. Denman, A. Okubo, T. Platt. The chlorophyll fluctuation spectrum in the sea. Limnol. Oceanogr., 22 (1977), 1033-1038.

[88] G. I. Taylor. The spectrum of turbulence. Proc. R. Soc. London Ser. A, 164 (1938), 476-490.

[89] L. Seuront, F.G. Schmitt. Eulerian and Lagrangian properties of biophysical intermittency in the ocean. Geophysical Research Letters, 31 (2004), L03306, doi:10.1029/2003GL018185.

[90] H. Yamazaki, D. Mackas, K. Denman. Coupling small scale physical processes with biology. The Sea: Biological-Physical interaction in the Ocean, edited by A.R. Robinson, J.J. McCarthy and B.J. Rothschild, Chapter 3: 51-112, 2002.

[91] H. Yamazaki, K. Squires. Comparison of oceanic turbulence and copepod swimming. Mar. Ecol. Prog. Ser., 144 (1996), 299-301.

[92] L. Seuront, H. Yamazaki, S. Souissi. Hydrodynamic disturbance and zooplankton swimming behavior. Zoological Studies, 43 (2004), 377-388.

[93] L. E. Karp-Boss, E. Boss, P. A. Jumars. Nutrient fluxes to planktonic osmotrophs in the presence of fluid motion. Mar. Biol. Ann. Rev., 34 (1996), 71-107.

[94] M. C. Gregg. Uncertainties and limitations in measuring $\epsilon$ and $\chi_{T}$. J. Atmos. Ocean. Tech., 16 (1990), 1483-1490. 
[95] I. N. McCave. Size spectra and aggregation of suspended particles in the deep ocean. DeepSea Res., 31 (1990), 329-352.

[96] T. Kirboe. Small-scale turbulence, marine snow formation, and planktivorous feeding. Sci. Mar., 61 (1997), 141-158.

[97] W. D. Gardner. The flux of particles to the deep sea: methods, measurements and mechanisms. Oceanography, 10 (1997), 116-121.

[98] G. Jackson, A. Bird. Aggregation in the marine environment. Environ. Sci. Tech., 32 (1998), 2805-2814.

[99] T. Kirboe, , K. P. Andersen, H. Dam. Coagulation efficiency and aggregate formation in marine phytoplankton. Mar. Biol., 107 (1990), 235-245.

[100] B. J. Rothschild, T. R. Osborn. Small-scale turbulence and plankton contact rates. J. Plankton Res., 10 (1988), 465-474.

[101] T. Kirboe, E. Saiz. Planktivorous feeding in calm and turbulent environment, with emphasis on copepods. Mar. Ecol. Prog. Ser., 122 (1995), 135-145.

[102] A. W. Visser, B. R. MacKenzie. Turbulence-induced contact rates of plankton: the question of scale. Mar. Ecol. Prog. Ser., 166 (1998), 307-310. 